Research Paper

\title{
Flow characteristic of highly underexpanded jets from various nozzle geometries
}

\author{
Xiaopeng $\mathrm{Li}^{\mathrm{a}, \mathrm{c}}$, Rui Zhou ${ }^{\mathrm{b}}$, Wei Yao ${ }^{\mathrm{c}}$, Xuejun Fan ${ }^{\mathrm{c}, *}$ \\ ${ }^{a}$ China Special Equipment Inspection and Research Institute, Beijing 100029, China \\ ${ }^{\mathrm{b}}$ Laboratory of Computational Physics, Institute of Applied Physics and Computational Mathematics, Beijing 100094, China \\ ' State Key Laboratory of High Temperature Gas Dynamics, Institute of Mechanics, Chinese Academy of Sciences, Beijing 100190, China
}

\section{H I G H L I G H T S}

- The effect of nozzle geometry on underexpanded jet flows is investigated using LES.

- Circular and square jets both correspond to a 3D helical instability mode.

- Elliptic and rectangular jets have a flapping instability in their minor axis plane.

- The formation and development of the intercepting shocks are highly different.

- The elliptic jet penetrates slowest, and has the largest overall mixing area.

\section{A R T I C L E I N F O}

\section{Article history:}

Received 9 December 2016

Revised 20 June 2017

Accepted 1 July 2017

Available online 03 July 2017

\section{Keywords:}

Underexpanded jets

Noncircular jets

Nozzle geometry

Large eddy simulation

\begin{abstract}
A B S T R A C T
Flow characteristics of highly underexpanded jets at the same nozzle pressure ratio of 5.60 but issuing from four different nozzles, i.e., the circular, elliptic, square, and rectangular nozzles, are studied using large eddy simulations. The results show that the square jet penetrates fastest, although the turbulence transition is similar for different jets. The penetration rates of different jets show the similar linear dependency on the square root of time, but the penetration constant $\Gamma$ for the noncircular jets deviates more than $5 \%$ from the theoretical value of 3.0. The circular and square jets both correspond to a three-dimensional helical instability mode, while the elliptic and rectangular jets have a two-dimensional flapping instability in their minor axis planes. All the jets undergo a Mach reflection forming the Mach disk, but the Mach disk in the elliptic and rectangular jets is not easily visible. The intercepting shocks in the square jet originate at the four corners of the nozzle exit at first, while the formation of the intercepting shocks is only observed in the major axis planes for the elliptic and rectangular jets. In addition, great differences are observed on the mixing characteristics between different jets. In particular, the elliptic jet penetrates slowest, has the shortest length of jet potential core, and takes the largest mixing area.
\end{abstract}

(c) 2017 Elsevier Ltd. All rights reserved.

\section{Introduction}

High-speed jets may be found in a great deal of practical applications, especially in energy and propulsion systems. A few of these include the injection of gaseous fuel jets in a natural gas engine [1,2] or a supersonic combustor [3,4], discharge of chimney gases into the atmosphere [5,6], and thrust control of rockets and $\mathrm{V} / \mathrm{STOL}$ aircraft via jets $[7,8]$. In most of these applications, an overall control of the fluid mechanics of the flow is required to improve the quality of the end process. Noncircular jets, such as the elliptic, square, and rectangular jets, are beneficial from this point of view

\footnotetext{
* Corresponding author.

E-mail addresses: lxpyfy@163.com (X. Li),xfan@imech.ac.cn (X. Fan).
}

by providing the efficient passive flow control through certain changes in nozzle geometry [9]. The flow of noncircular jets becomes even more complicated when they operate at underexpanded conditions, when the nozzle exit pressure is higher than the ambient one and the jet structures are characterized by the quasi-periodic shocks. Therefore, the study of underexpanded noncircular jets will be of great value for both engineering applications and gas dynamics.

Motivated by the possible advantages on the mixing and entrainment rates, numerous studies have been conducted on underexpanded noncircular jets, with most research being primarily experimental. For example, Rajakuperan and Ramaswamy [10] investigated experimentally the elliptic jets for various nozzle pressure ratios (NPR) up to 20.3, and found the barrel shocks were 
only presented in the major axis plane of the jets. Mitchell et al. [11] presented the mean and fluctuating velocity profiles in underexpanded elliptic jets with an aspect ratio (AR) of 2.0 at NPR from 2.2 to 4.2 using planar particle image velocimetry (PIV). Chauhan et al. [12] studied the mixing characteristics of underexpanded elliptic jets at NPR from 2.0 to 5.0 based on the pressure measurements. Grinstein et al. $[13,14]$ investigated the near-field dynamics and turbulence transition in square jets. Gutmark et al. [15] and Raman [16,17] concentrated on studies of the acoustic properties and shock structures in underexpanded rectangular jets. Behrouzi and Mcguirk [18] investigated the flow development in supersonic underexpanded jets issuing from a rectangular nozzle using schlieren visualization, pitot probe, and laser doppler anemometry measurements. Valentich et al. [19] examined the mixing characteristics of a moderate aspect ratio supersonic rectangular jet using PIV measurements. In addition, Rao and Jagadeesh [20] conducted measurements to examine two novel supersonic nozzles, and found a $30 \%$ increase in entertainment and mixing based on the laser scattering flow visualization.

Gutmark et al. [21,22] compared the mixing characteristics between the circular, elliptic and rectangular jets from the subsonic flow to supersonic flow, and found that the elliptic and rectangular jets had a higher spreading rate relative to the circular jet, especially at the minor axis plane. After that, there have been some efforts [23-28] devoted to directly comparing the flow properties between the underexpanded jets issuing from different nozzle geometries. For example, Otobe et al. [23] studied the influences of the nozzle geometry on the Mach disk height and diameter in highly underexpanded jets by solving the two-dimensional, axisymmetric Euler equations. Zare-Behtash et al. [24,25] explored the effect of nozzle geometry on the initial shock and vortex development in supersonic underexpanded jets using high-speed schlieren photography and PIV measurements. Menon and Skews [26] reported the near-field shock structures of underexpanded sonic jets issuing from square, rectangular, elliptic, and slot nozzles based on schlieren photographs and Reynolds-averaged NavierStokes (RANS) simulations. Xu et al. [27] compared the near-field mean and fluctuating velocity field of five different noncircular jets using PIV measurements. Zhang et al. [28] investigated the initial flow differences between the supersonic underexpanded circular and square jets based on large eddy simulation. Note that most of these studies focused on the differences of near-field shock structures $[23,26]$ or the initial flow development $[24,25,28]$ between the jets. In the case of obtaining an indepth and comprehensive understanding on the noncircular jets, a detailed comparisons of their various flow characteristics are thus necessary.

There are several numerical studies on underexpanded noncircular jets, see for example the RANS modeling on square jets by Tsutsumi et al. [29] and the RANS simulations performed by Menon and Skews [26] and Yang et al. [30]. These RANS based simulations mainly provided the time-averaged flow properties, but leaving the unsteady flow characteristics of the jets unresolved. Recently, large eddy simulation (LES), which resolves the large scales directly and models the effect of small scales, has been used to explored the unsteady flow characteristics of underexpanded jets. For example, Vuorinen et al. [31] performed LES modeling of underexpanded jets at different NPR, and further compared the flow differences between the underexpanded methane and nitrogen jets [32]. Hamzehloo and Aleiferis [33] focused on the flow characteristics of the underexpanded hydrogen jets, and then compared the mixing characteristics of hydrogen jets with the methane jets at various NPR [34]. Li et al. [35] investigated the differences of flow structures and screech characteristics between the underexpanded hydrogen and nitrogen jets using LES, and also explored the turbulence transition mechanism and three-dimensional instability of underexpanded jets at different NPR [36]. These studies show that the LES technique is capable of capturing the salient flow structures and dynamics in supersonic underexpanded jets. However, most of these LES studies focused on the axisymmetric circular jets. Revealing the unsteady flow physics of underexpanded jets issuing from different nozzles using high-resolution LES modeling is therefore of great benefit for practical mixing enhancement design and active flow control.

In the present study, three-dimensional large eddy simulations of highly underexpanded jets at the same nozzle pressure ratio of 5.60 but issuing from different nozzle geometries are carried out. The circular nozzle with an exit diameter of $d=2.0 \mathrm{~mm}$ is set as the baseline case. Three other nozzles, i.e., the elliptic, square, and rectangular nozzles, are employed to investigate the effect of the nozzle geometry on the flow characteristics. A highresolution, hexahedral, and block-structured grid containing about 31.8 million computational cells is applied. The compressible flow solver, astroFoam, which is developed based on the OpenFOAM C++ library, is used to perform the simulations. The flow evolution and jet penetration, quasi-steady state jets, near-field shock structures, and mixing characteristics for different jets are discussed and compared in detail.

\section{Computational methodology}

\subsection{Governing equations and numerical methods}

The governing equations used in the present LES are threedimensional filtered compressible Navier-Stokes equations, including the following conservation equations for mass, momentum, energy, and species:

$$
\begin{aligned}
& \frac{\partial \bar{\rho}}{\partial t}+\frac{\partial \bar{\rho} \tilde{u}_{i}}{\partial x_{i}}=0 \\
& \frac{\partial \bar{\rho} \tilde{u}_{i}}{\partial t}+\frac{\partial \bar{\rho} \tilde{u}_{i} \tilde{u}_{j}}{\partial x_{j}}=-\frac{\partial \bar{p}}{\partial x_{i}}+\frac{\partial \tilde{\tau}_{i j}}{\partial x_{j}}-\frac{\partial \tau_{i j}^{s g s}}{\partial x_{j}} \\
& \frac{\partial \tilde{\rho} \tilde{h}_{s}}{\partial t}+\frac{\partial \tilde{\rho} \tilde{u}_{j} \tilde{h}_{s}}{\partial x_{j}}=\frac{\partial \bar{p}}{\partial t}+\frac{\partial \tilde{u}_{j} \tilde{\tau}_{i j}}{\partial x_{i}}-\frac{\partial \bar{q}_{i}}{\partial x_{i}}-\frac{\partial H_{i}^{s g s}}{\partial x_{i}} \\
& \frac{\partial \bar{\rho} \tilde{Y}_{k}}{\partial t}+\frac{\partial \bar{\rho} \tilde{u}_{j} \tilde{Y}_{k}}{\partial x_{j}}=\frac{\partial}{\partial x_{j}}\left(\bar{\rho} \tilde{D}_{k m} \frac{\partial \tilde{Y}_{k}}{\partial x_{j}}\right)-\frac{\partial \Phi_{k, j}^{s g s}}{\partial x_{j}}
\end{aligned}
$$

where $\rho$ is the density, $u_{i}$ is the velocity in $x_{i}$ direction, $p$ is the pressure, $\tau_{i j}$ is the viscous stress tensor, $h_{s}$ is the sensible enthalpy, $q_{i}$ is the heat flux vector, $Y_{k}$ is the mass fraction, $D_{k m}$ is the equivalent binary mass diffusivity. The pressure is computed from the equation of state:

$\bar{p}=\bar{\rho} R \tilde{T}$

where $\tilde{T}$ is the temperature, $R$ is the gas constant of the mixture gas, $R_{u}$ is the universal gas constant. The terms with superscript sgs in equations (1)-(4) denote sub-grid quantities. In particular, the SGS energy fluxes term, $H_{i}^{\text {sgs }}=\left(\bar{\rho} \widetilde{E_{t} u_{i}}-\bar{\rho} \tilde{E}_{t} \tilde{u}_{i}\right)+\left(\overline{p u_{i}}-\bar{p} \tilde{u}_{i}\right)$, is modeled based on a linear eddy diffusivity assumption as:

$H_{j}^{s g s}=-\bar{\rho} \frac{v_{t}}{P r_{t}} \frac{\partial \tilde{H}}{\partial x_{j}}=-\bar{\rho} \frac{v_{t}}{P r_{t}}\left(\frac{\partial \tilde{h}}{\partial x_{j}}+\tilde{u}_{i} \frac{\partial \tilde{u}_{i}}{\partial x_{j}}+\frac{\partial k^{s g s}}{\partial x_{j}}\right)$

where $v_{t}$ is the eddy viscosity. The SGS species fluxes term, $\Phi_{k, j}^{s g s}=\bar{\rho} \widetilde{u_{j} Y_{k}}-\bar{\rho} \tilde{u}_{j} \tilde{Y}_{k}$, is also modeled using the eddy diffusivity assumption as:

$\Phi_{k, i}^{s g s}=-\bar{\rho} \frac{v_{t}}{S c_{t}} \frac{\partial \tilde{Y}_{k}}{\partial x_{i}}$ 
Generally, one-equation model has advantages on modeling the transitional flows or flows with large scale unsteadiness compared to the "zero" equation models (e.g. Smagorinsky model). Our previous work [36] also indicated that the one-equation model is recommended if focusing on the acoustic properties of underexpanded jets. Therefore, the SGS stress term, $\tau_{i j}^{s g s}=\bar{\rho}\left(\widetilde{u_{i} u_{j}}-\tilde{u}_{i} \tilde{u}_{j}\right)$, is modeled with the sub-grid scale turbulent kinetic energy one-equation model [37] as:

$\frac{\partial \bar{\rho} k^{s g s}}{\partial t}+\frac{\partial \bar{\rho} \tilde{u}_{j} k^{s g s}}{\partial x_{j}}=\frac{\partial}{\partial x_{j}}\left[\bar{\rho}\left(\frac{v_{t}}{P r_{t}}+v\right) \frac{\partial k^{s g s}}{\partial x_{j}}\right]-\tau_{i j}^{s g s} \frac{\partial \tilde{u}_{i}}{\partial x_{j}}-C_{\varepsilon} \frac{\bar{\rho}\left(k^{s g s}\right)^{3 / 2}}{\bar{\Delta}}$

$v_{t}=C_{v} \bar{\Delta} \sqrt{k^{s g s}}$

where $C_{v}$ and $C_{\varepsilon}$ are model constants. The thermodynamic and transport properties of individual species, such as the enthalpy per unit mass $h_{k}$ and the specific heat at constant pressure $c_{p k}$, are calculated based on NIST-JANAF thermo-physical and transport database [38]. The dynamic viscosity $\mu_{k}$ is computed by Sutherland's law.

The density-based compressible solver astroFoam, which is developed based on the open-source CFD (Computational Fluid Dynamics) package OpenFOAM 2.3.0, is used to solve the above equations. The astroFoam solver is well established and validated in our previous LES of underexpand hydrogen [35] and nitrogen [36] jets issuing from circular nozzles, thus only the main aspects of the astroFoam solver are described here for concision. In astroFoam, the convection-diffusion equation is solved by semi-discrete KT (Kurganov and Tadmor) scheme [39] for shock capturing and turbulence resolving. NVD (Normalised Variable Diagram) scheme based on the Minmod limiter [40] is applied to reconstruct the primitive values at faces to obtain the second order accuracy. Time integration is carried out by the Crank-Nicholson scheme [41], which is second order accurate.

\subsection{Computational geometry and grid}

The computational domain and nozzle geometries used in the present LES are similar to that presented in our previous studies [35,36], and Fig. 1 shows the schematic of computational model for the circular jet as well as the coordinate system for an example. The computational domain mainly consist of a box of size $50 \times 100 \times 50 \mathrm{~mm}$ in $\mathrm{x}-, \mathrm{y}-$, and $\mathrm{z}$-directions, respectively. The nitrogen jet (mass fraction $\mathrm{Y}_{\mathrm{N} 2}=1.0$ ) in the high-pressure nozzle (with total pressure $P_{0}$, and total temperature $T_{0}$ ) is injected into the quiescent air (with stastic pressure $P_{\infty}$, and stastic temperature $\mathrm{T}_{\infty}$ ) from a convergent nozzle. The high-pressure nozzle has a height of $20.0 \mathrm{~mm}$ and is divided into three sections in the streamwise direction. The first section is a cylinder with a height of $10.0 \mathrm{~mm}$ and a diameter of $8.0 \mathrm{~mm}$. The third section is $4.0 \mathrm{~mm}$ in height, and has a cross section of circle, ellipse, square, and rectangle, respectively, for different jets studied. The second section provides a smooth transition from the first section to the third section over a length of $6.0 \mathrm{~mm}$. The nozzle exit area is equal for different jets, and the circular nozzle has an exit diameter of $2.0 \mathrm{~mm}$. The aspect ratios (AR) for the elliptic and rectangular jets are both 2.0. The schematic of all the nozzles is illustrated in Fig. 2, and their dimensions are given in Table 1 in detail.

As indicated by the previous studies [31-36,42-44], the spatial resolution in LES of supersonic jets needs to be rather high. A grid independence study by using two different meshes, i.e. the coarse and fine meshes, is performed in our previous work [36], and it is found that the fine mesh has advantages on capturing the turbulence characteristics and acoustic properties of the underexpanded jets. Therefore, the meshes used in the present LES are designed referring the previous "fine mesh" in Ref. [36], as shown in Fig. 3. The meshes are hexahedral, block-structured, and contain about 31.8 million cells. The gird around the nozzle exit, the jet potential core, and the jet shear is specially refined to obtain high resolution. With these careful arrangements, the grid resolution in the main region of interest in this study is similar as that used in the
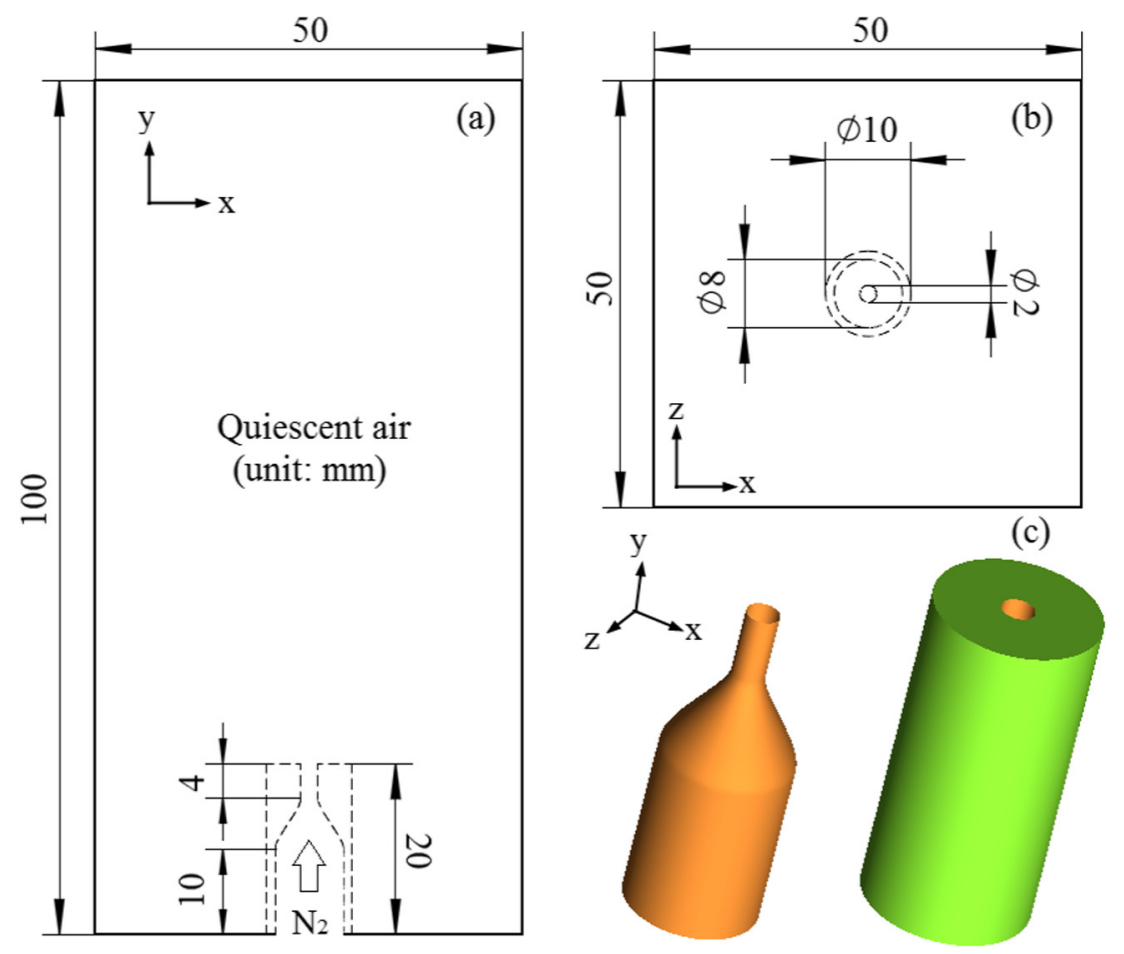

Fig. 1. Schematic of the computational model for the circular jet. (a) Cross streamwise view, (b) top view, (c) three-dimensional schematic of the nozzle. 


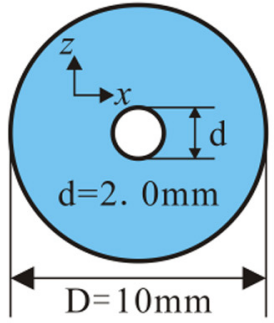

(a) Circle

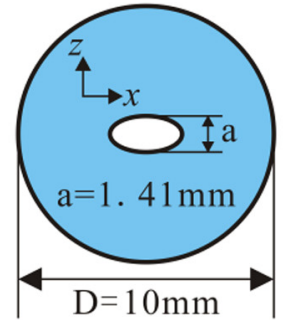

(b) Ellipse

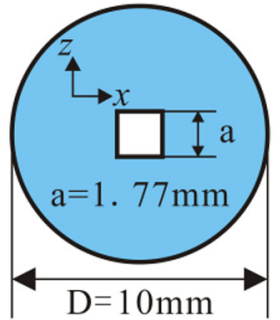

(c) Square

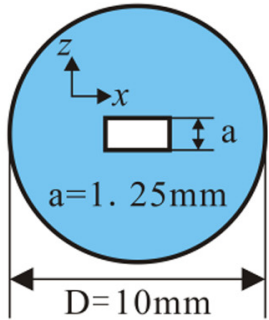

(d) Rectangle

Fig. 2. Nozzle geometries used for LES of underexpanded jets.

Table 1

Dimensions of the nozzles.

\begin{tabular}{llll}
\hline Nozzle & Aspect ratio $(\mathrm{AR})$ & Major axis $(\mathrm{mm})$ & Minor axis $(\mathrm{mm})$ \\
\hline Circle & 1.0 & 2.00 & 2.00 \\
Ellipse & 2.0 & 2.82 & 1.41 \\
Square & 1.0 & 1.77 & 1.77 \\
Rectangle & 2.0 & 2.50 & 1.25 \\
\hline
\end{tabular}

previous LES of supersonic jets [31-34,42-44], which are summarized in Table 2. On the other hand, coarse cell sizes with a resolution of $1.0 \mathrm{~mm}$ in the far field and $0.5 \mathrm{~mm}$ at outflow boundaries are used to avoid wave reflections by introducing additional dissipation.

\subsection{Initialization and boundary conditions}

The quiescent air is the mixture of $76.699 \%$ nitrogen and $23.301 \%$ oxygen in mass fraction. Initially the temperature, pressure, and velocity of the ambient air are all uniform, i.e. $\mathrm{T}_{\infty}=300 \mathrm{~K}, \mathrm{P}_{\infty}=101,325 \mathrm{~Pa}, \mathrm{U}_{\infty}=0$. The nitrogen gas in the highpressure nozzle with different export section shape is injected into the quiescent air at the same stagnation temperature of $360 \mathrm{~K}$ and the same total pressure of $0.57 \mathrm{MPa}$, resulting in the same nozzle pressure ratio (NPR) of 5.60 for different jets. The flow conditions at the nozzle exit are close to sonic conditions, and are summarized in Table 3 in detail. During the simulations, the stagnation condition for temperature and pressure is employed at the high-pressure nozzle inlet, while the velocity is treated with a zero-gradient condition. All walls are treated as no-slip, adiabatic

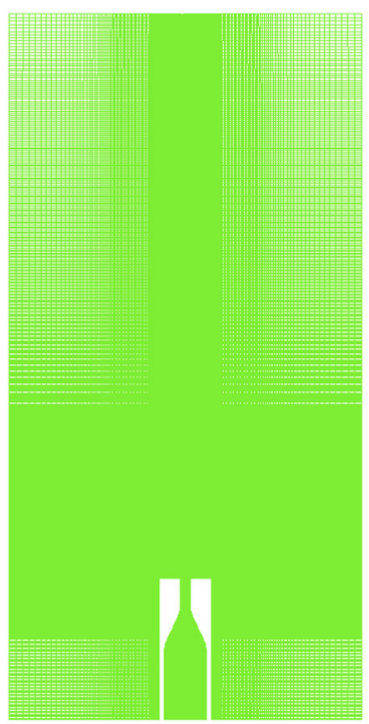

(a)

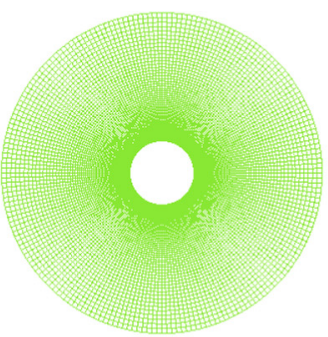

(b)

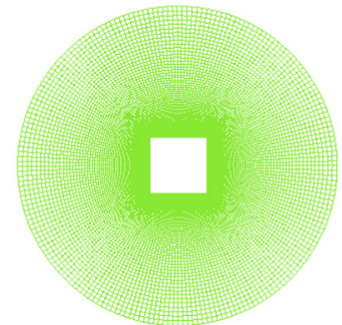

(d)

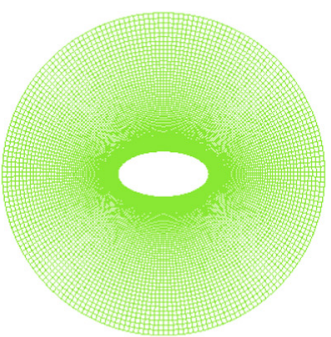

(c)

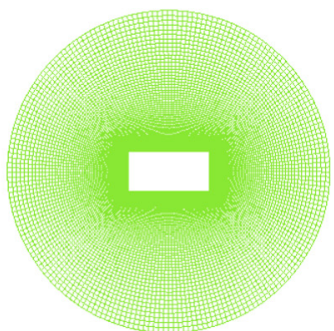

(e)

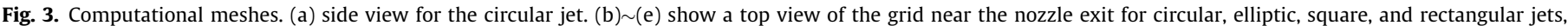
successively.

Table 2

Grid resolution comparison in the near field (r/D: -1.5 to $1.5 ; \mathrm{y} / \mathrm{D}: 0-5 \mathrm{D})$ of supersonic jets.

\begin{tabular}{|c|c|c|c|c|c|c|c|}
\hline Grid & $\mathrm{D}(\mathrm{mm})$ & $\Delta \mathrm{r}_{\min }$ & $\Delta \mathrm{r}_{\max }$ & $\Delta \mathrm{y}_{\min }$ & $\Delta y_{\max }$ & $\operatorname{Re}$ & Total $\left(\times 10^{6}\right)$ \\
\hline Present Work & 2.0 & $\mathrm{D} / 200$ & $\mathrm{D} / 52$ & $\mathrm{D} / 67$ & $\mathrm{D} / 25$ & $\sim 10^{5}$ & 27.3 \\
\hline Vuorinen et al. $[31,32]$ & 1.4 & $\mathrm{D} / 70$ & $\mathrm{D} / 50$ & $\mathrm{D} / 35$ & $\mathrm{D} / 25$ & $\sim 10^{5}$ & 12.0 \\
\hline Hamzehloo and Aleiferis [33,34] & 1.5 & $\mathrm{D} / 50$ & $\mathrm{D} / 50$ & $\mathrm{D} / 50$ & $\mathrm{D} / 25$ & $\sim 10^{5}$ & 13.5 \\
\hline Gorle et al. [42] & 2.0 & $\mathrm{D} / 100$ & $\mathrm{D} / 50$ & $\mathrm{D} / 100$ & $\mathrm{D} / 25$ & $\sim 10^{5}$ & 17.4 \\
\hline Dauptain et al. [43] & 25.4 & $\mathrm{D} / 35$ & $\mathrm{D} / 30$ & $\mathrm{D} / 35$ & $\mathrm{D} / 30$ & $\sim 10^{6}$ & 22.0 \\
\hline Rana et al. [44] & 4.0 & $\mathrm{D} / 33$ & $\mathrm{D} / 33$ & $\mathrm{D} / 33$ & $\mathrm{D} / 33$ & $\sim 10^{4}$ & 9.2 \\
\hline
\end{tabular}


Table 3

Jet exit flow conditions at the sonic orifice.

\begin{tabular}{llll}
\hline Property & Symbol & Value & Unit \\
\hline Mach number at nozzle exit & $\mathrm{M}_{1}$ & 1.0 & - \\
Stagnation pressure & $\mathrm{P}_{0}$ & 0.57 & $\mathrm{MPa}$ \\
Stagnation temperature & $\mathrm{T}_{0}$ & 360 & $\mathrm{~K}$ \\
Velocity at nozzle exit & $\mathrm{U}_{1}$ & 353.1 & $\mathrm{~m} / \mathrm{s}$ \\
Fully expanded jet Mach number, & $M_{j}$ & 1.78 & - \\
Reynolds number at nozzle exit & $R e_{1}$ & 1.36 & $\times 10^{5}$ \\
Nozzle pressure ratio (NPR) & $\mathrm{P}_{0} / \mathrm{P}_{\infty}$ & 5.60 & - \\
Nozzle density ratio (NDR) & $\rho_{1 / \rho_{\infty}}$ & 2.87 & - \\
\hline
\end{tabular}

conditions. At the top of the computational domain and at the four free surfaces, an open boundary condition is used, i.e. all flow parameters are treated as zero-gradient for outflow and fixed ambient values in case of backflows.

The computational time step ranges between $\triangle \mathrm{t}=1.0 \times 10^{-8} \mathrm{~S}$ and $\triangle \mathrm{t}=1.4 \times 10^{-8} \mathrm{~s}$ for the four different jets studied, all of which are limited by the same maximum Courant-Friedrichs-Lewy (CFL) number of 0.6. The flow-through time (FTT) for the jets washing out the computational domain in streamwise direction is around $0.5 \mathrm{~ms}=200 \mathrm{t}_{0}$, with $\mathrm{t}_{0}=2.5 \times 10^{-6} \mathrm{~s}=2.5 \mu \mathrm{s}$. The total simulation duration is thus set as $4 \mathrm{FTT}=2.0 \mathrm{~ms}=800 \mathrm{t}_{0}$. The instantaneous results are saved every $2 t_{0}$, and turbulent statistics are collected for the last three flow-through times (3FTT, 200t $\sim 800 \mathrm{t}_{0}$ ).

\section{Results and discussion}

\subsection{Jet evolution and penetration}

The time evolutions of nitrogen mass fraction for circular and elliptic jets are depicted in Fig. 4. As can be seen, the flow evolution of elliptic jet at different time is similar to the circular jet. For example, the initial flow field for both jets on either $\mathrm{ZY}$ or $\mathrm{XY}$ planes is well symmetric with respect to the jet centerline and characterized by a tip vortex ring, which has been previously observed in supersonic underexpanded jets $[24,25]$ as well as in subsonic jets $[45,46]$. The turbulence transitions of the circular and elliptic jets are all characterized by the breakdown of recirculation zones, the loss of flow symmetry, and the generation of streamwise vortexes, as stated in our previous study [36]. Meanwhile, the large-scale turbulent vortices, which have also been identified in previous numerical modeling of supersonic underexpanded jets [31-36], are both visible along the jet shear layer when the jets are fully developed. Fig. 4 also shows that the flow structures on the ZY plane are similar to that on the XY plane for the circular jet. However, the flow structures in the minor (ZY) and major (XY) axis planes are different for the elliptic jet. Firstly, the tip vortex ring in the initial stage is not symmetric to the jet centerline for the elliptic jet. Secondly, the jet boundary in the major axis plane for the fully developed elliptic jet decreases at first, and then increases its width with increasing streamwise distance, which is different from the fact that the width of jet boundary generally increases along the streamwise direction. It's also noticed that the elliptic jet appears to spread to a bigger zone in the minor axis plane than in the major axis plane.

In addition, Fig. 4 indicates that the elliptic jet penetrates faster than the circular jet initially, but its penetration rate gets slower than the circular jet when the jets are fully developed. Fig. 5(a) presents the jet penetration $z(t)$ as a function of time, which quantitatively characterizes the flow evolution of different jets. Note that the jet penetration $z(t)$ is defined according to the outer limit of nitrogen mass fraction on the midline plane [36], i.e. the maximum axial position as shown in Fig. 4. Fig. 4 also suggests the jet penetration computed according to the minor axis plane is equal to that calculated based on the major axis plane. From Fig. 5(a), the jet penetration of different jets is close to each other when $t / t_{0}<80$ (in the first two stages, i.e. the initial stage and turbulence transition stage), but notable differences are observed after $t / t_{0}=100$. In particular, the square jet penetrates fastest, and has a shortest FTT time of around $160 \mathrm{t}_{0}$. The elliptic jet

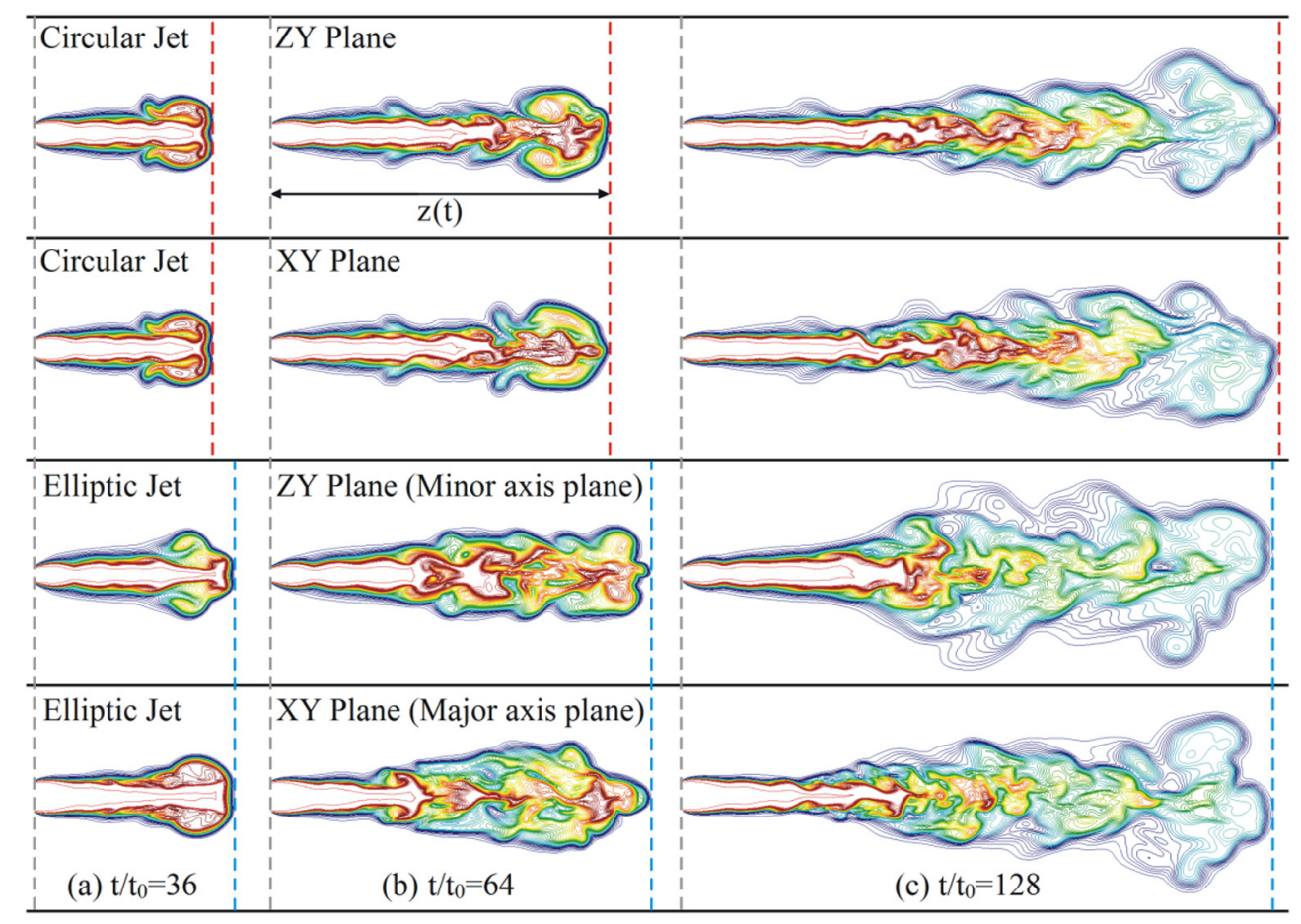

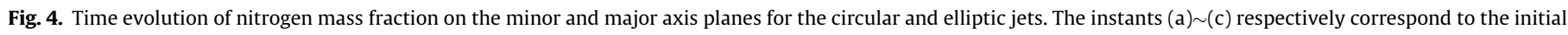
phase, the transition phase, and the fully developed flow. 

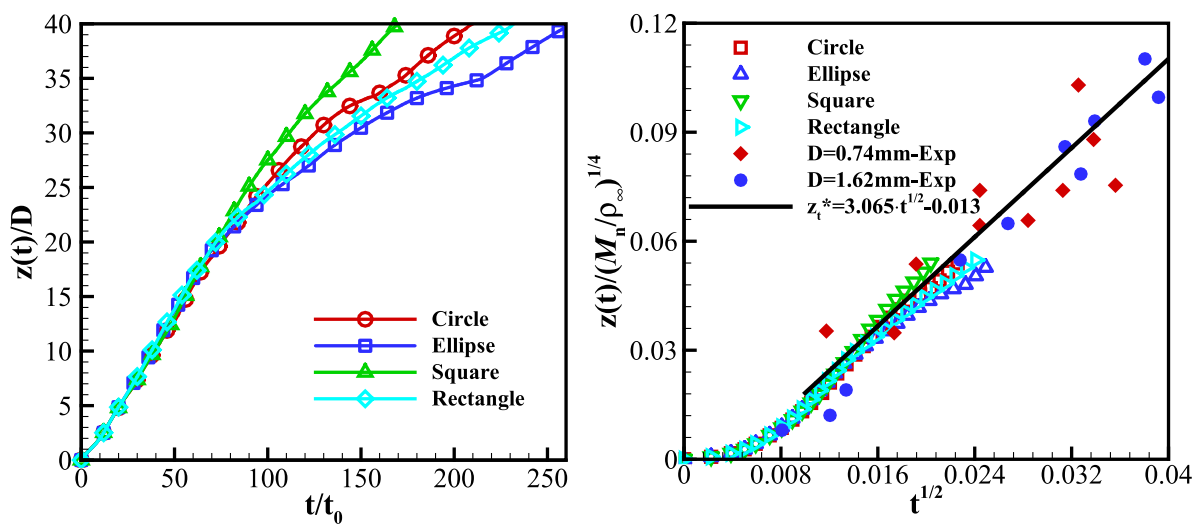

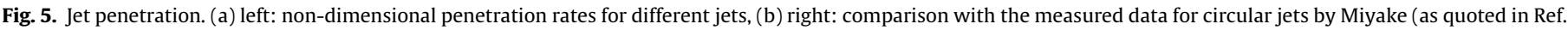
[48]).

penetrates slowest, and its FTT time is approaching to $260 t_{0}$. The circular and rectangular jets penetrate faster than the elliptic jet but slower than the square jet, and take a FTT time of about $210 \mathrm{t}_{0}$ and $230 \mathrm{t}_{0}$, respectively.

Hill and Ouellette $[47,48]$ found that the penetration of the fuel jets issued from round nozzles obeys a linear dependency on the square root of time based on measurements, and further derived a formula to predict the penetration of underexpanded circular jets as:

$\frac{z(t)}{\left(\dot{M}_{n} / \rho_{\infty}\right)^{1 / 4} t^{1 / 2}}=\Gamma$

where $z(t)$ is the jet penetration, $\dot{M}_{n}$ is the momentum injection rate, $t$ is the time from the beginning of injection, $\Gamma$ is a constant equal to $3.0 \pm 0.1$. Note that Eq. (10) can be transformed directly to the following form [48]:

$\frac{z(t)}{d_{e q}}=\Gamma\left(\frac{\pi}{4}\right)^{1 / 4}\left(\frac{t U_{1}}{d_{e q}}\right)^{1 / 2}$

where $d_{e q}$ is the equivalent diameter $d_{e q}=D\left(\rho_{1} / \rho_{\infty}\right)^{1 / 2}, \rho_{1} / \rho_{\infty}$ is the nozzle density ratio (NDR), and $U_{1}$ is the velocity at the nozzle exit.

Fig. 5(b) presents the predicted jet penetration normalized according to Eqs. (6)-(10) in comparison with the experimental data by Miyake (as quoted in Ref. [48]), while the jet penetration constants $\Gamma$ for different jets are listed in Table 4 . As can be seen, the penetration rates of different jets show the similar linear dependency on the square root of time after the initial turbulence transition. In particular, the penetration constant $\Gamma$ of the circular jet predicted by the present LES takes a value of 2.946 . This value is close to the theoretical value of 3.0, and is also in good agreement with the measured data of 3.065 in spite of the slight differences in NDR. However, the penetration constants of the elliptic, square and rectangular jets are $2.485,3.336$, and 2.736 , respectively, which deviate more than $5 \%$ from the theoretical value of 3.0 for the circular jet. These differences are mainly due to the inherent differences in flow evolution of jets issuing from different nozzle geometries. This observation suggests that the jet penetration constant $\Gamma$ is not only affected by NDR (or NPR) $[36,48]$, but is also strongly influenced by the nozzle geometries used.

\subsection{Quasi-steady state jets}

The instantaneous density gradient after the jets are fully developed in both the minor and major axis planes is presented in Fig. 6, which shows the global flow structures for different jets. For the circular jet, the general flow patterns in both planes are very similar, and some of the typical characteristics of underexpanded jets are evident. For example, the near-field shock structures show a wavy up and down oscillation, and increase in oscillation amplitude progressively for the shocks formed further downstream from the nozzle exit, as visualized using schlieren photographs by Panda [49]. The inner shear layer (slip lines) persists at about the fourth shock cell, and then merges with the outer shear layer (jet boundary), which is accordance with the measurement by Mitchell et al. [50]. The acoustic waves that originate around the end of jet potential core propagate both upstream and downstream [51]. It is also noticed that the jet boundary expands along the streamwise direction from the end of the jet potential core in both planes for the circular jet.

Fig. 6 also indicates that the key flow features of the square jet, including the shock structures, the acoustic patterns, and the jet boundary, are similar to the circular jet. Considering the symmetry of the flow patterns in the minor and major axis planes, only the minor axis planes are depicted hereafter for the circular and square jets. On the other hand, the flow characteristics of the elliptic jet are similar to the rectangular jet, but differ greatly from the circular and square jets. In particular, the flow patterns in the minor axis plane for the elliptic and rectangular jets are not similar to the major axis plane any more, and some differences are evident. For the elliptic and rectangular jets, the shocks in the minor axis plane oscillate wavily with larger amplitude than the circular jet, but the

Table 4

Comparison of jet penetration constants.

\begin{tabular}{|c|c|c|c|c|c|c|}
\hline Author & Technique & Nozzle & $\mathrm{D}(\mathrm{mm})$ & NDR & $\Gamma$ & $\Delta \Gamma$ \\
\hline Hill and Ouellette [47] & Theory & Circle & - & - & $3.0 \pm 0.1$ & $\pm 3.33 \%$ \\
\hline Miyake (quoted in Ref. [48]) & Experiment & Circle & 0.741 .62 & 3.2 & 3.065 & $2.17 \%$ \\
\hline \multirow[t]{4}{*}{ Present work } & LES & Circle & 2.00 & 2.87 & 2.946 & $-1.80 \%$ \\
\hline & & Ellipse & - & 2.87 & 2.485 & $-17.17 \%$ \\
\hline & & Square & - & 2.87 & 3.336 & $11.20 \%$ \\
\hline & & Rectangle & - & 2.87 & 2.736 & $-8.80 \%$ \\
\hline
\end{tabular}


(a)

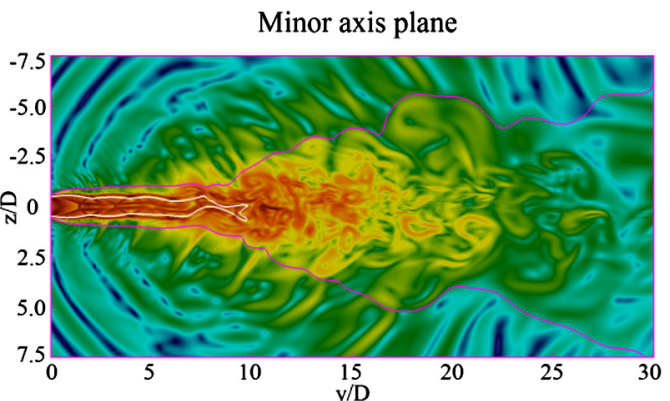

(b)

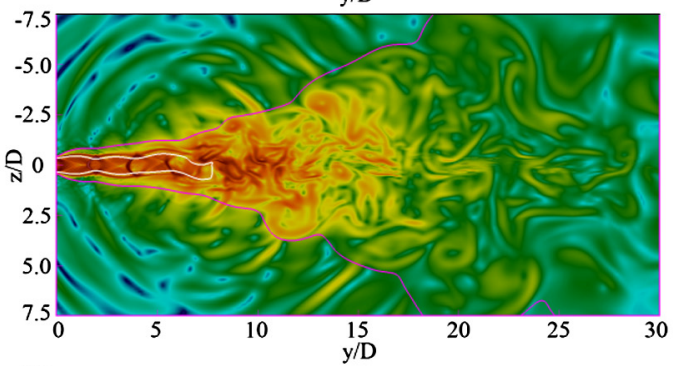

(c)

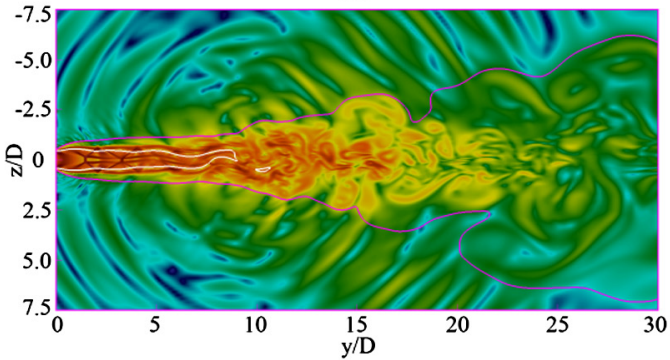

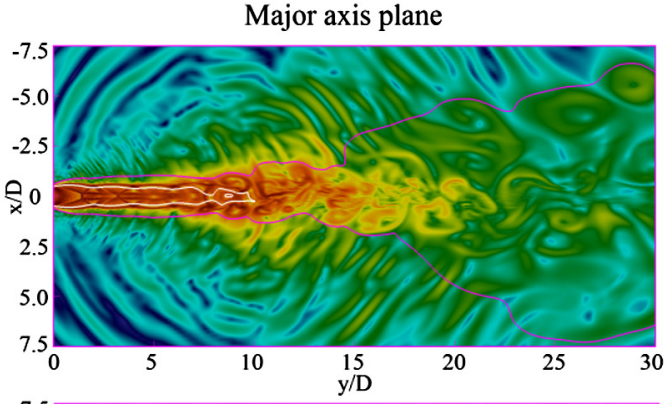
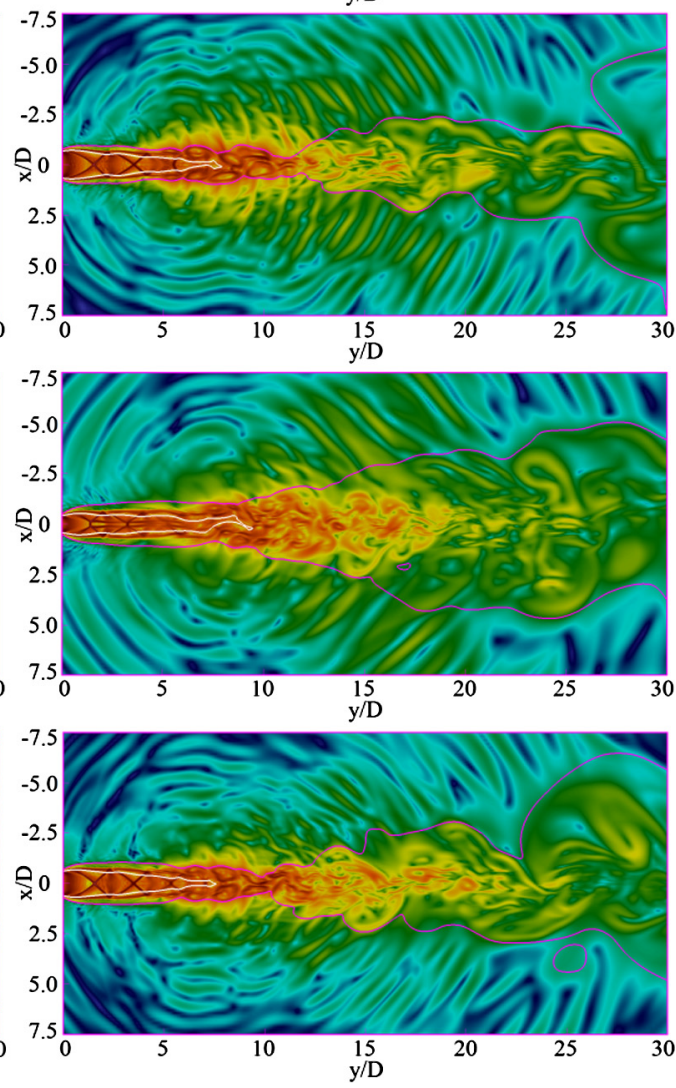

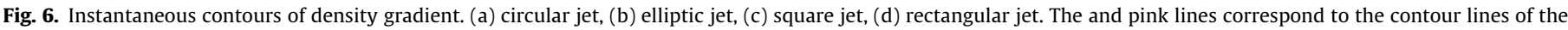

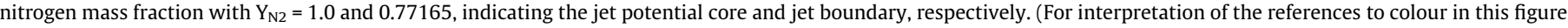
legend, the reader is referred to the web version of this article.)

shocks and jet potential core in the major axis plane appear to be steady and symmetric to the jet centerline. Meanwhile, the jet boundary of the elliptic and rectangular jets expands extremely in the minor axis plane, significantly faster than for the circular and square jets. In contrast, the jet boundary of the elliptic and rectangular jets in the major axis plane shrinks at first, and then expands along the streamwise direction. This behavior is mainly contributed to the axis switching effect that has been previously observed in the noncircular jets, including the square [13,14,29], elliptic [10-12], and rectangular jets [18,19]. Generally, the axis switching angle for the square jet is $45 \mathrm{deg}$, but takes a value of $90 \mathrm{deg}$ for the elliptic and rectangular jets. It is thus reasonable that the elliptic and rectangular jets spread more quickly and toward a much larger region in the minor axis plane.

The spatial structure and dynamics of vortex are usually examined in previous studies to characterize the axis switching effects in non-circular jets. Fig. 7 compares the vortex structures of different jets resolved by present LES in terms of the iso-surfaces of the Q-criterion. The topology structures of these vortexes are extremely complex, and are three-dimensional, especially in the downstream region. In the near-field region (i.e. at $y / D<8$ ), the large scale vortex pockets in the circular and square jets are alternately distributed along the jet shear layer, and can be observed both in the minor and major axis planes. This suggests that the circular and square jets are in three-dimensional helical instability modes. On the other hand, the alternating pockets of large scale shear layer vortices in the near-field region of elliptic and rectangular jets only exist in the minor axis planes. The presence of these alternating vortex pockets in the minor axis plane but their absence in the major axis plane clearly indicates that the large scale instability for the elliptic and rectangular jets is twodimensional in nature; there is a flapping instability in the minor axis plane for these two jets. Fig. 8 presents the pressure fluctuations sampled on either side of the jet for the circular and elliptic jets. It is evident that the pressure fluctuations on either side of circular jet appear to be correlated and in phase opposition in both planes. However, the phase of the two pressure signals in elliptic jet is opposite in minor axis plane, but is consistent in the major axis plane. These findings further confirm the conclusions that in the near-field region the circular jet corresponds to a 

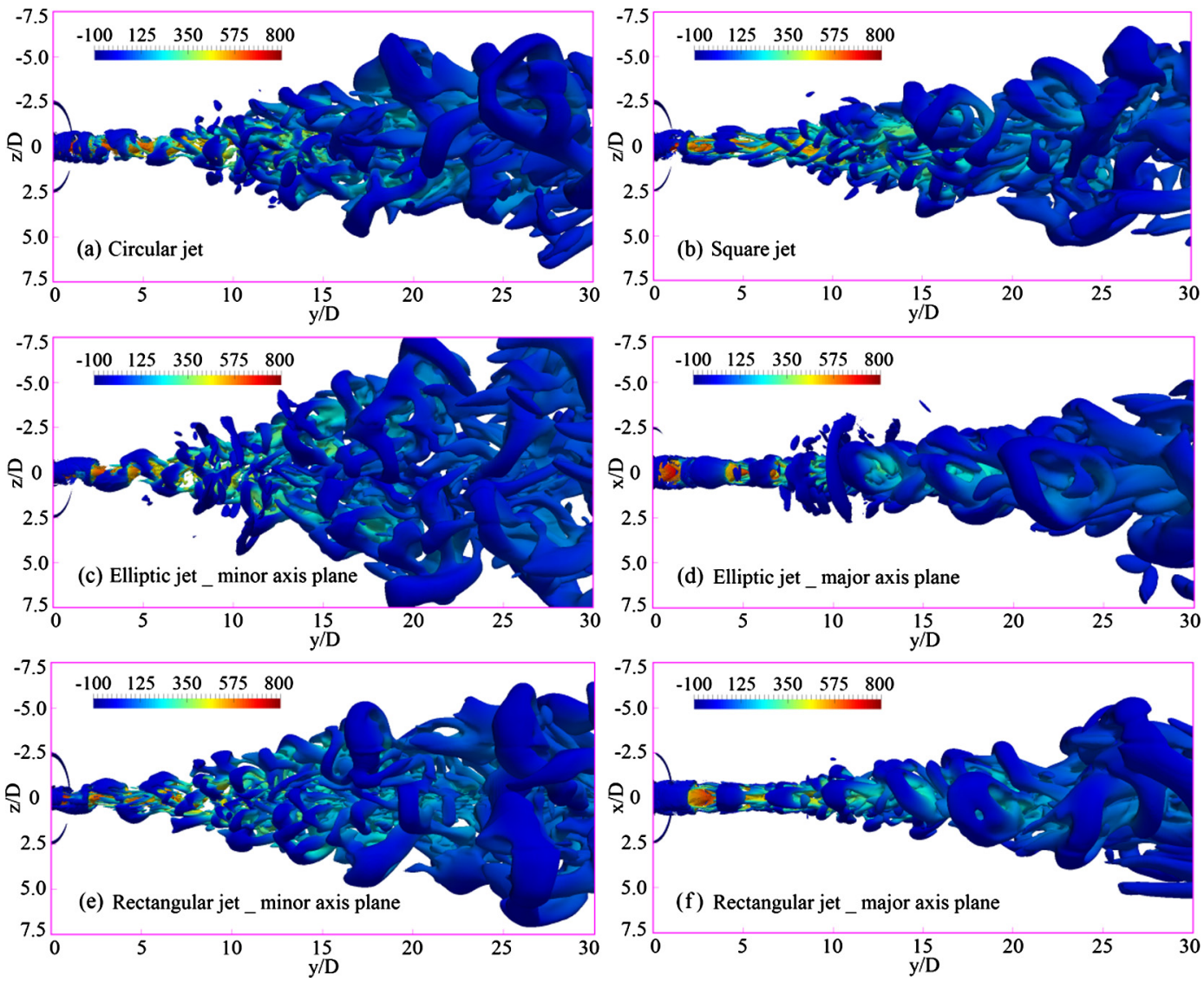

Fig. 7. Three-dimensional iso-surfaces of the $\mathrm{Q}$-criterion $\left(\mathrm{Q}=10^{8} \mathrm{~s}^{-2}\right)$ colored by streamwise velocities for different jets.
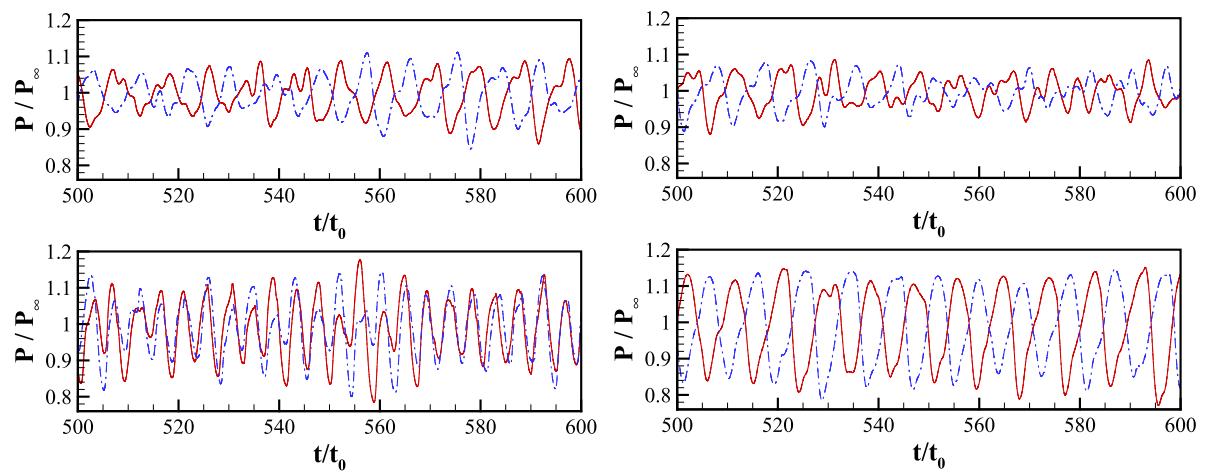

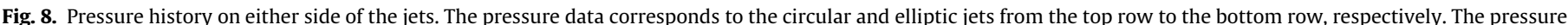

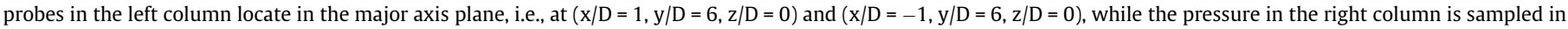
the minor axis plane, i.e., at $(x / D=0, y / D=6, z / D=1)$ and $(x / D=0, y / D=6, z / D=-1)$.

three-dimensional helical instability mode but the elliptic jet is in a two-dimensional instability mode. Fig. 8 also shows the pressure fluctuations in the elliptic jet are higher than in the circular jet, which quantitatively demonstrates the previous observation based on Fig. 6 that the shocks in the minor axis plane of the elliptic jet oscillate at much larger amplitude than in the circular jet.

In addition, Mitchell et al. [11] found the velocity fluctuations are much stronger in the major axis plane of elliptic jets at NPR between 2.2 and 4.2 based on PIV measurements, and concluded that significantly higher mixing is taking place there. However, Hussain and Husain [52] found significantly higher levels of mass-velocity fluctuations in the minor axis plane of an ideally expanded supersonic elliptical jet. Gutmark et al. [21] also found the higher intensity of near-field pressure fluctuations on the minor axis plane for the supersonic underexpanded elliptic jets experimentally. Fig. 8 indicates that the pressure fluctuations near the jet centerline in both planes are similar for elliptic jet. On the other hand, the alternating passage of vortices in the minor axis planes of the elliptic and rectangular jets induce the intense oscillation motion of shocks inside the jets and the rapid outward displacement of the jet boundary as shown in Fig. 6, which will further lead to the higher mixing between the jet and its surroundings there.

\subsection{Near nozzle shock structures}

Fig. 9(a) shows the time-averaged near-nozzle shock structures of the circular jet. It is evident that the standard shock structures in 

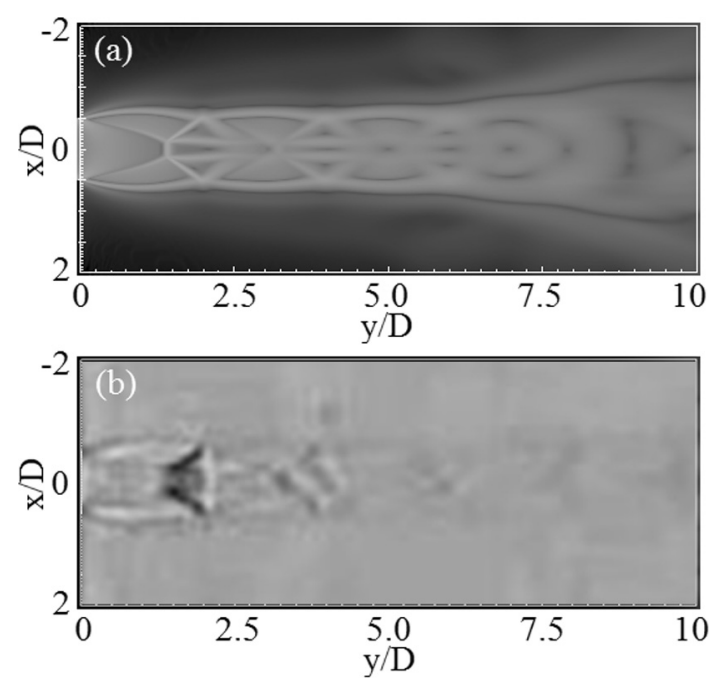

Fig. 9. Comparison of time-averaged near-field shock structures in circular jets between the LES prediction and experiment. (a) density gradient obtained by LES, (b) schlieren photography.

the near field of a highly underexpanded jet, including the Mach disk, intercepting shock, triple point, reflected shock and slip lines [50,53], are all well captured by the present LES. Meanwhile, the general show structures predicted by LES compare reasonably with the schlieren photography shown in Fig. 9(b) at the same NPR of 5.60 .

In the recent review on highly underexpanded circular jets, Franquet et al. [53] indicated that the height of Mach disk is mainly governed by NPR and weakly influenced by the exit nozzle angle. On the other hand, the width of the Mach disk is governed by NPR, but is strongly dependent on the nozzle geometry and shape. It have also been reported that Mach disk dimensions of underexpanded jets are affected by the level of turbulence at the nozzle exit, and higher turbulence level may result in larger Mach disk dimensions [54]. Fig. 10 quantitatively compares the timeaveraged density of the circular jets between the present LES results for NPR $=5.60$ and the measured data by Panda and Seasholtz [55] at a similar NPR of 5.74. A good agreement with the axial profiles of experimental data is observed, especially for the first shock position, i.e., the Mach disk height. This observation further confirms the conclusion by Franquet et al. [53] that the Mach disk height of underexpanded jets is dominated by NPR. At the first two streamwise positions of $\mathrm{y} / \mathrm{D}=0.9$ and 1.2 , the radial profiles of density predicted by LES also agree well with the measured data. However, the predicted density peaks at around $x / D=0.2$ at $y / D=1.5$, which is smaller than the measured value of $x / D=0.25$. This finding suggests that the current LES reproduces a narrower
Mach disk than the experiment. Similarly, the LES studies performed by Vuorinen et al. [31] and Hamzehloo and Aleiferis [54] also predicted a smaller Mach disk diameter compared with the measurements. The contributing factors, as one would expect, can be the differences in nozzle geometry design and simulation setup (eg. the turbulence levels at the nozzle exit) between the LES and experiment.

Generally, the circular jet is considered as highly underexpanded when NPR increases beyond 3.85 [56], when the regular reflection of the intercepting shock can no longer happen on the jet centerline and a normal shock (i.e., the Mach disk) appears instead. However, it is still vague when a Mach reflection happens for the rectangular or elliptic jets. For example, the schlieren photographys by Raman and Taghavi [16] shows no Mach disk for $\mathrm{Mj}=1.75$ (corresponding to a nozzle pressure ratio of NPR $=5.32$ ) in an underexpanded rectangular jet with $A R=4.0$. Valentich et al. [19] also found no Mach reflection for NPR $=4.86$ and $\mathrm{Mj}=1.69$ in a rectangular jet with AR of 4.0 based on PIV (Particle Image Velocimetry) measurements. For the elliptic nozzle with AR of 2.0, Menon and Skews [26] din not observe a Mach reflection for NPR $=3.8$ based on RANS simulations, but observed an apparent Mach disk at NPR $=5.7$. Mitchell et al. [11] found experimentally the appearances of Mach reflection from NPR $=2.6$ onwards in elliptic jet with $A R=2.0$. However, the Mach disk is only evident at NPR of 5.0 in the schlieren photographys by Chauhan et al. [12] in the underexpanded elliptic jet with AR of 2.0. Fig. 11 compares the near-nozzle shock structures in different planes for different jets obtained by the present LES. It is evident that the intercepting shocks in the square jet propagate downstream and reflect in an irregular manner as the circular jet, forming a Mach reflection and a three-shock system consisting of the intercepting shock, the Mach disk, and the reflected shock. The elliptic and rectangular jets undergo a Mach reflection as well, but the Mach disk is not easily visible due to its size. The presence of the Mach reflection for the elliptic and rectangular jets can be inferred from the slip lines emanating from reflection point. On the other hand, the slip lines are not easily observed in the major axis planes as compared to the minor axis planes for the elliptic and rectangular jets, which suggests that the Mach disk on the minor axis plane of the nozzle is larger than in the major axis plane. This finding shows that the cross-section of the Mach disk has switched axes with $90 \mathrm{deg}$ as compared to the axes of the nozzle exit, which is in accordance with the experimental observations on elliptic jets by Menon and Skews [26] and Mitchell et al. [11].

Besides the width of the Mach disk, the prominent differences between the near-field shock structures of jets are the presence of the intercepting shocks and their origins. Fig. 11 shows that the origin of the intercepting shocks in circular jet is near the nozzle exit, while the intercepting shocks along the major axis plane of the square jet are observed to occur some distances downstream of
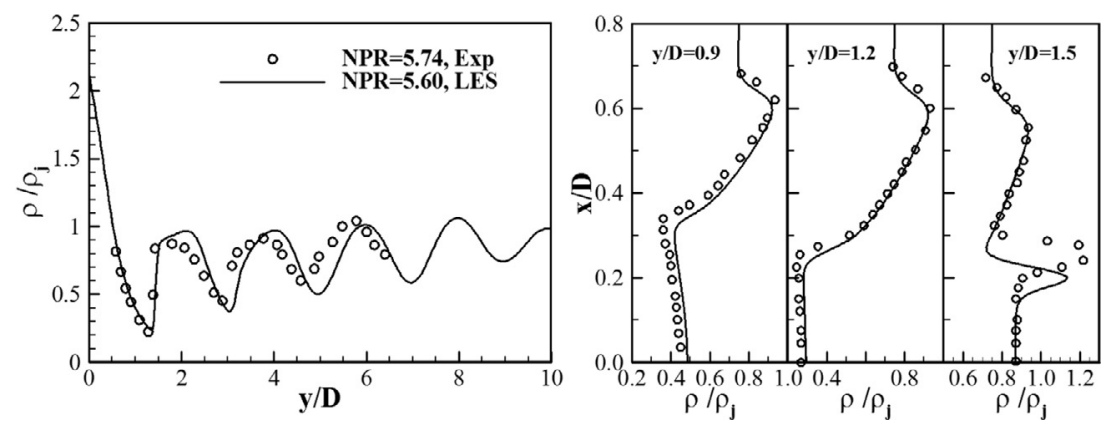

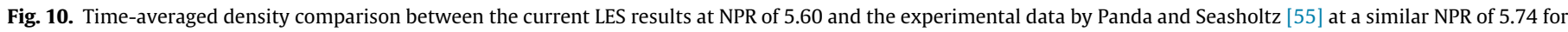
circular jets. (a) left: axial profile, (b) right: radial profiles at different streamwise positions. 
(a)
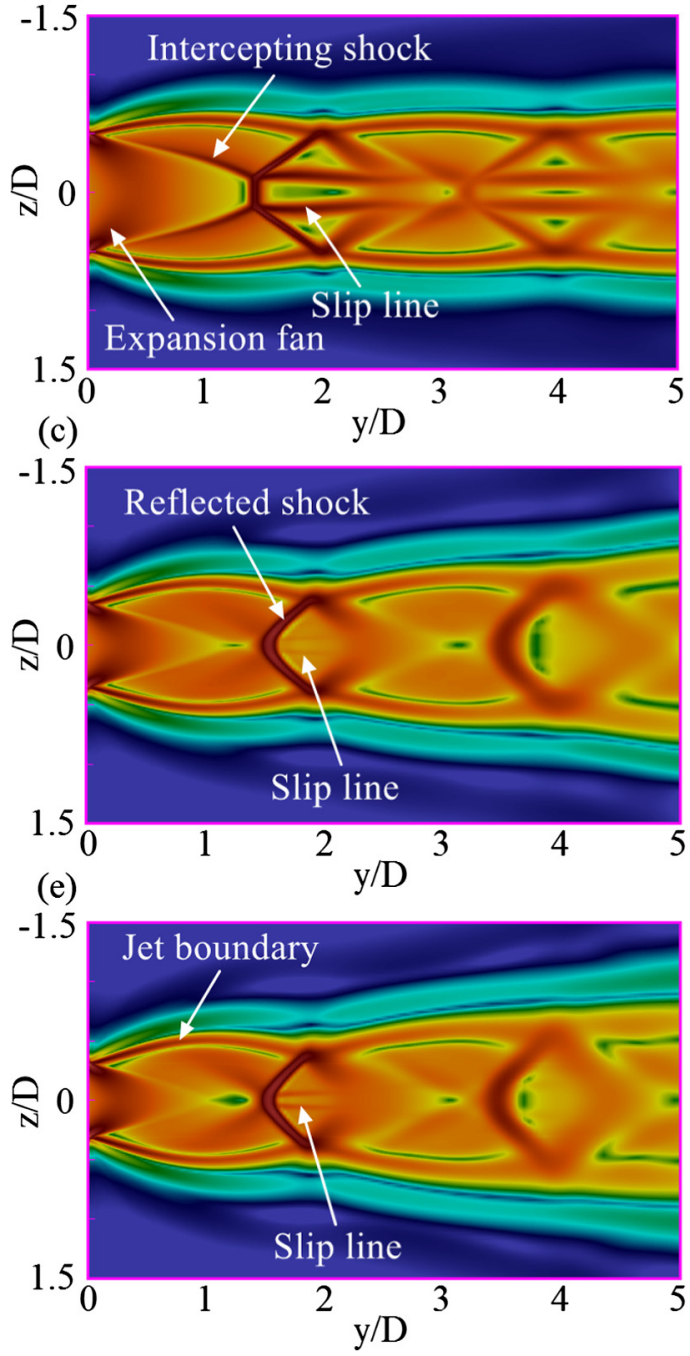

(b)

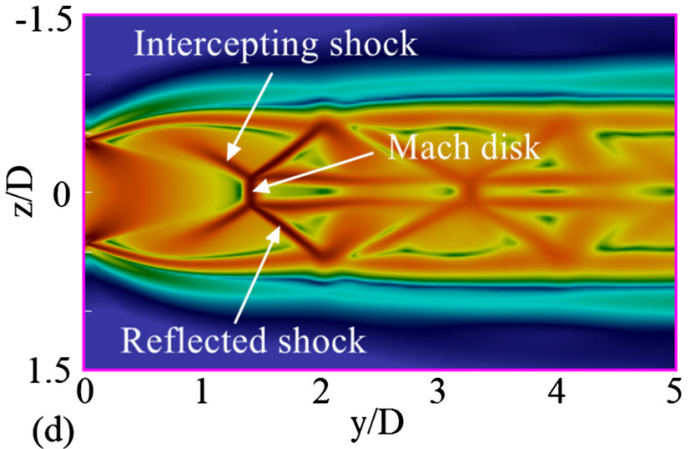

(d)

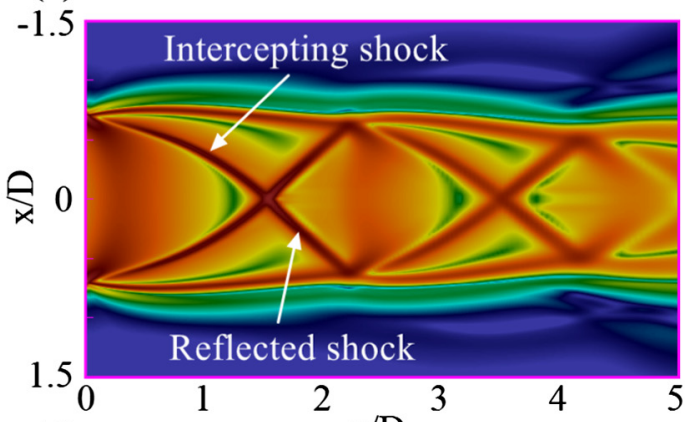

(f)

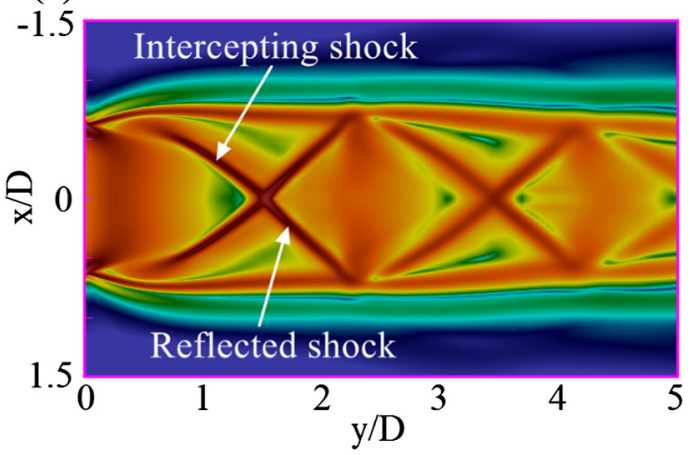

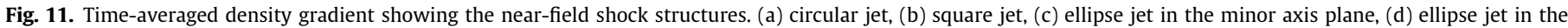
major axis plane, (e) rectangular jet in the minor axis plane, (f) rectangular jet in the major axis plane.

the nozzle exit. On the other hand, the formation of the intercepting shock is observed in the major axis planes but is lacking in the minor axis planes for both the elliptic and rectangular jets. Note that the presence of the intercepting shocks in the major axis plane and the absence of them in the minor axis plane have been also observed experimentally by Verma and Rathakrishnan [57] and Menon and Skews [26] on elliptic jets.

Fig. 12 shows the three-dimensional near-field shock structures for different jets, where the formation and development of the intercepting shocks is evident. As can be seen, the jet boundary, the intercepting shock surface, and the reflected shock surface in the circular jet are well symmetrical with respect to the jet centerline and appear to be circular in cross-section. On the other hand, the intercepting shocks in the square jet originate from the four corners of the nozzle exit at first, and then are observed along the major axis plane some distance downstream of the nozzle exit. For the elliptic jet, the intercepting shocks originate near the nozzle exit, and are mainly located along the major axis plane. The intercepting shocks in the rectangular jet are mainly observed along the major axis plane as the elliptic jet, but originate at the four corners of the nozzle as the square jet at first. As a result, the intercepting shocks in the rectangular jets are observed farer downstream of the nozzle exit than in the elliptic jet in the 2D slices of the major axis planes, as shown in Fig. 11(d) and (f).

The mean profiles of nitrogen mass fraction $Y_{N 2}$ shown in Fig. 13 (a) indicate that the elliptic and rectangular jets have a similar jet potential core length of about 7D, which is shorter than the $8 \mathrm{D}$ for circular and square jets. Meanwhile, the elliptic jet takes the smallest $Y_{N 2}$ values at different streamwise positions after the jet potential core, while the $Y_{N 2}$ values for square jet is largest. This suggests that a better mixing between the injected gas and its surrounding is taking place for the jet issued from the elliptic nozzle. Fig. 13(b) presents the time-averaged profiles of pressure along the jet centerline for different jets. As can be seen, the pressure decreases sharply from the high nozzle exit value, oscillates around a high level, increases to a maximum at the first shock (i.e. the Mach disk), and approaches to the ambient values from above the end of jet potential core. In particular, the positions of the first three shocks are similar for different jets, which can also be observed from the previous Fig. 11. Meanwhile, the strength of the first two shocks in the elliptic and rectangular jets is close to each other, but is much larger than that in the circular and square jets, which is characterized by a more intense jump in pressure 


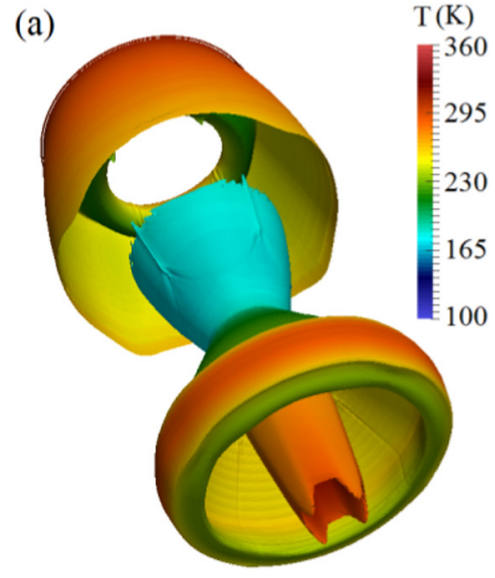

(b)

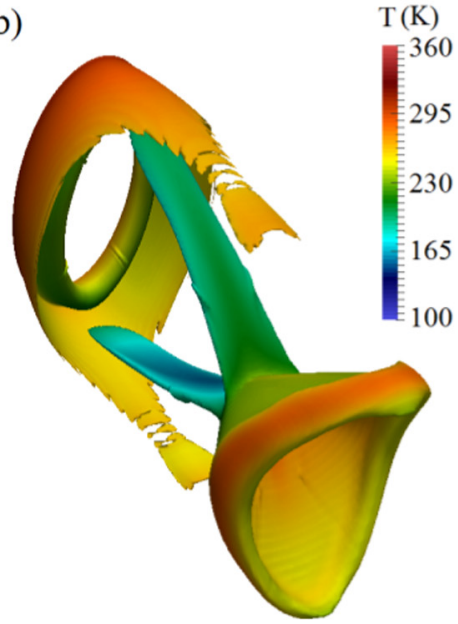

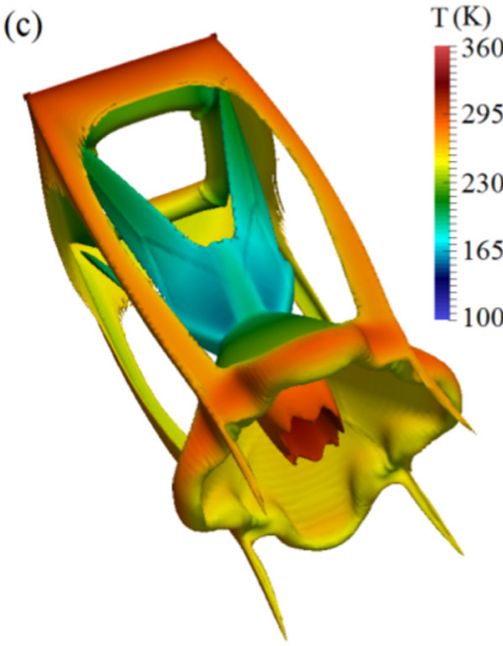

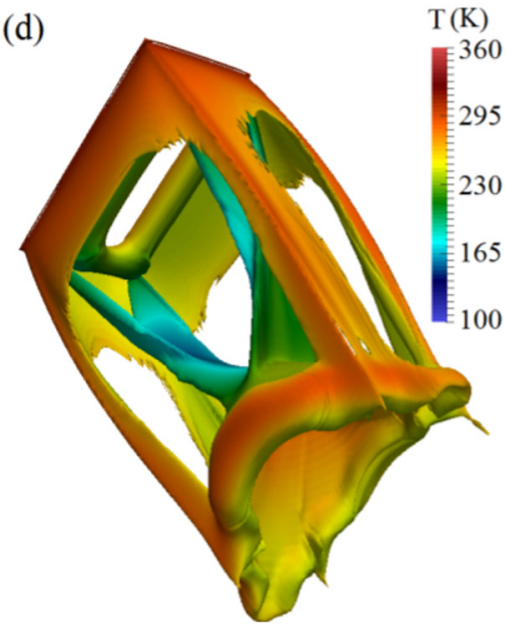

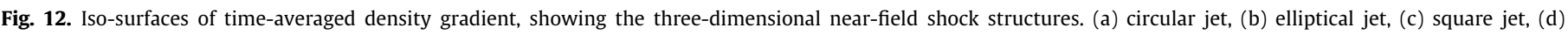
rectangular jet.
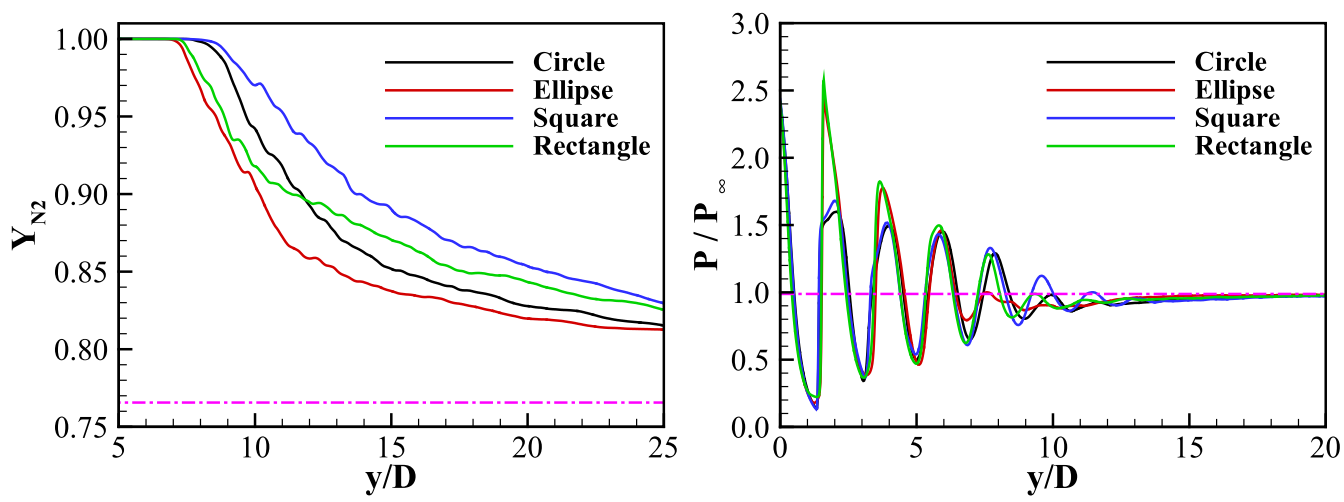

Fig. 13. Time-averaged flow properties along the jet centerline. (a) left: mass fraction of nitrogen, (b) right: non-dimensional pressure.

values after those shocks. On the other hand, the pressure peak at the fourth shock for the elliptic jet is relatively smaller when compared to other jets, which suggests that the pressure in elliptic jet decreases to the ambient value more quickly.

\subsection{Mixing characteristics}

The differences in flow structures (for example, as indicated by the previous Figs. 6, 7 and 11) will result in the different mixing characteristic. The jet mixing performances are quantitatively evaluated based on the mixing area on cross-section planes in this section. Fig. 14(a) shows the contour lines of nitrogen mass fraction on the cross-section plane of $\mathrm{y} / \mathrm{D}=14$ for the ellipse jet, and the area covered by the $\mathrm{Y}_{\mathrm{N} 2}=0.77165$ contour line (pink thick line) is defined as the mixing area $A$. Note that the nitrogen mass fraction $\mathrm{Y}_{\mathrm{N} 2}=0.77165$ corresponds to a mixture fraction level of $Z=0.02$. The time history of non-dimensional mixing area, i.e. the mixing area normalized by the nozzle exit area $A^{*}$, on the cross-section 

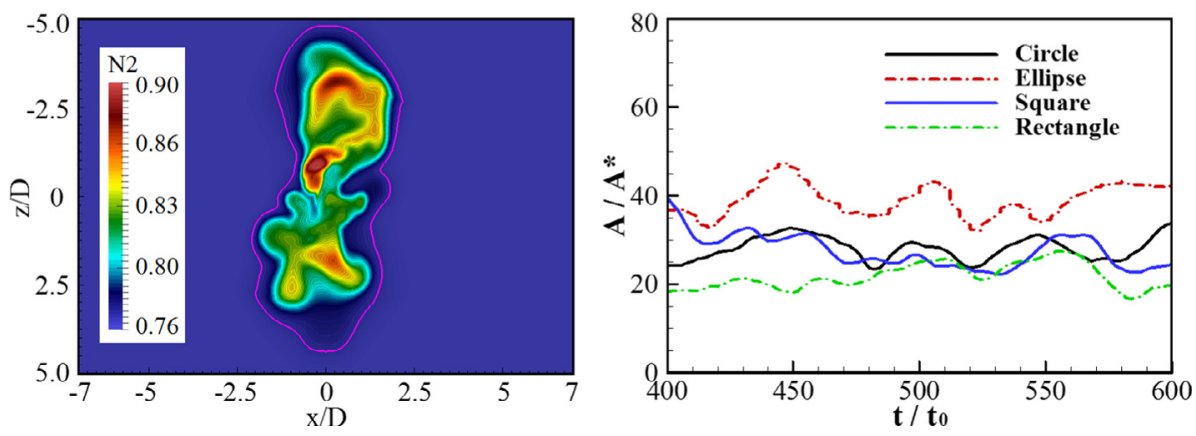

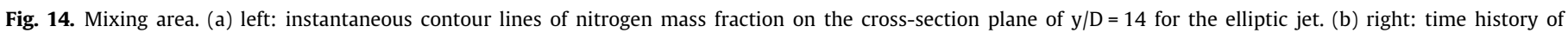
normalized mixing area on the cross-section plane of $y / D=14$ for different jets.

plane of $y / D=14$ for different jets is shown in Fig. 14(b). As can be seen, the instantaneous mixing area shows some low-frequency oscillations, instead of being a constant. This behavior is probably connected with the development of large scale coherent structures along the jet shear layer, as shown in Figs. 6 and 7. It is also noticed that the mixing areas of circular, square and rectangular jets are close to each other at this location (i.e. at $\mathrm{y} / \mathrm{D}=14$ ), but are all smaller than the elliptic jet.

The time-averaged mixing area on cross-section planes is plotted as a function of streamwise positions for different jets in Fig. 15 (a). As expected, the overall trend of mixing area profile is similar for different jets, i.e., the mixing area is relatively small in the nearfield region, and increases quickly from around the end of jet potential core as the jets spread toward the radial direction (see, for example Figs. 6 and 7). Meanwhile, the mixing area of different jets is close to each other in the near nozzle region. In the downstream of $y / D>8$, generally the elliptic jet has the largest mixing area, and the mixing area of the square jet is smallest, while the mixing area of rectangular jet is larger than the square jet but smaller than the circular jet.

Fig. 15(b) shows the axial profiles of relative mixing area, $\Delta A=\left(A-A_{\text {circle }}\right) / A_{\text {circle }}$, which evaluates how much the mixing area of different jets deviates from the circular jet. It is interesting to find that the $\Delta A$ for the elliptic, square, and rectangular jets almost all reaches the maximum values at around the end of jet potential core. The $\Delta A$ for the square and rectangular jets decreases along the streamwise direction after $\mathrm{y} / \mathrm{D}=8$, and reaches values below zero after around $y / D=10$, then approaches to a similar value of $-26 \%$ at $y / D=30$. On the other hand, the $\Delta A$ for the elliptic jet appears to have an equal value of around 25\% at different streamwise
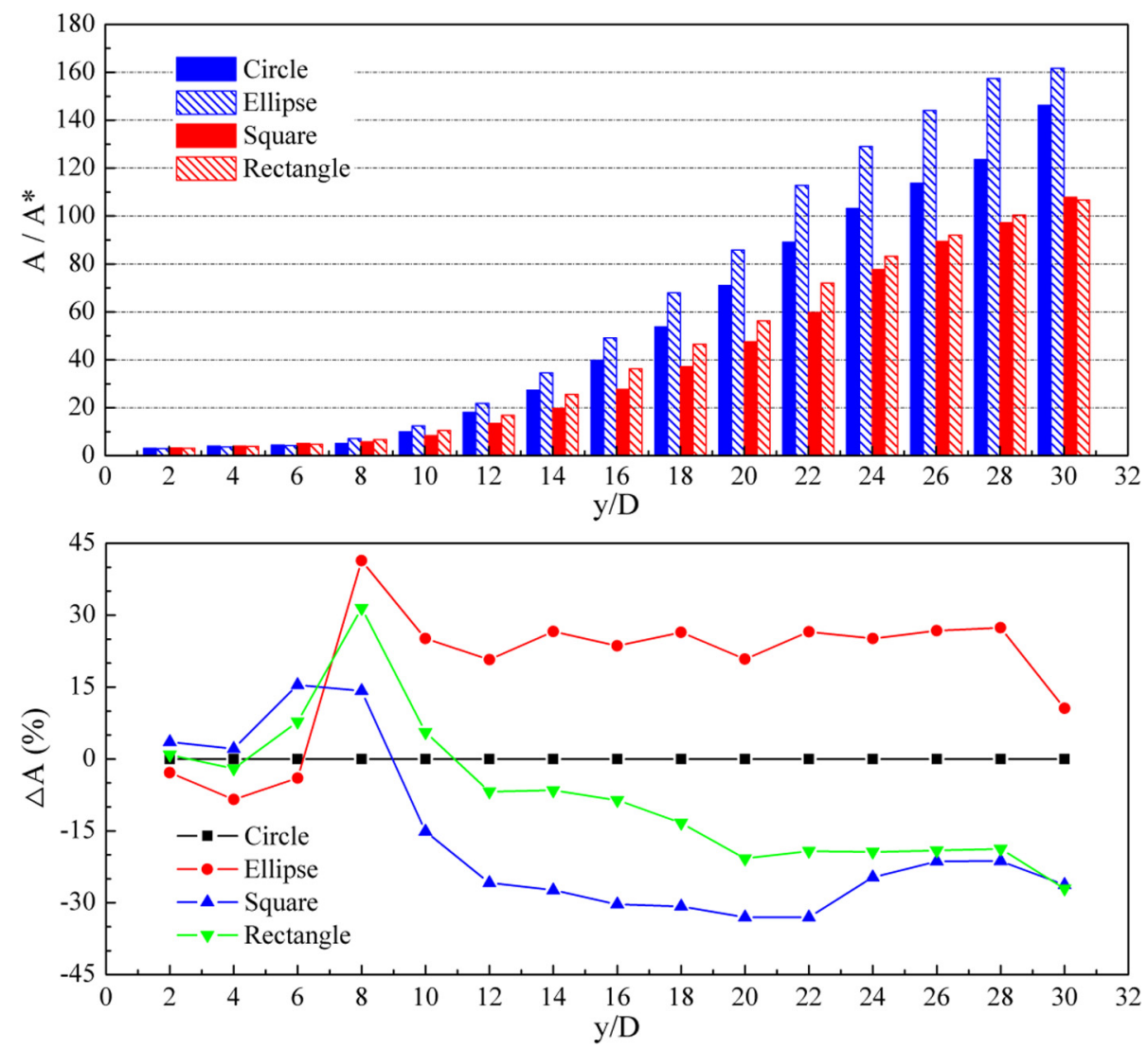

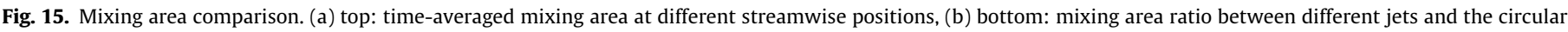
jet. 

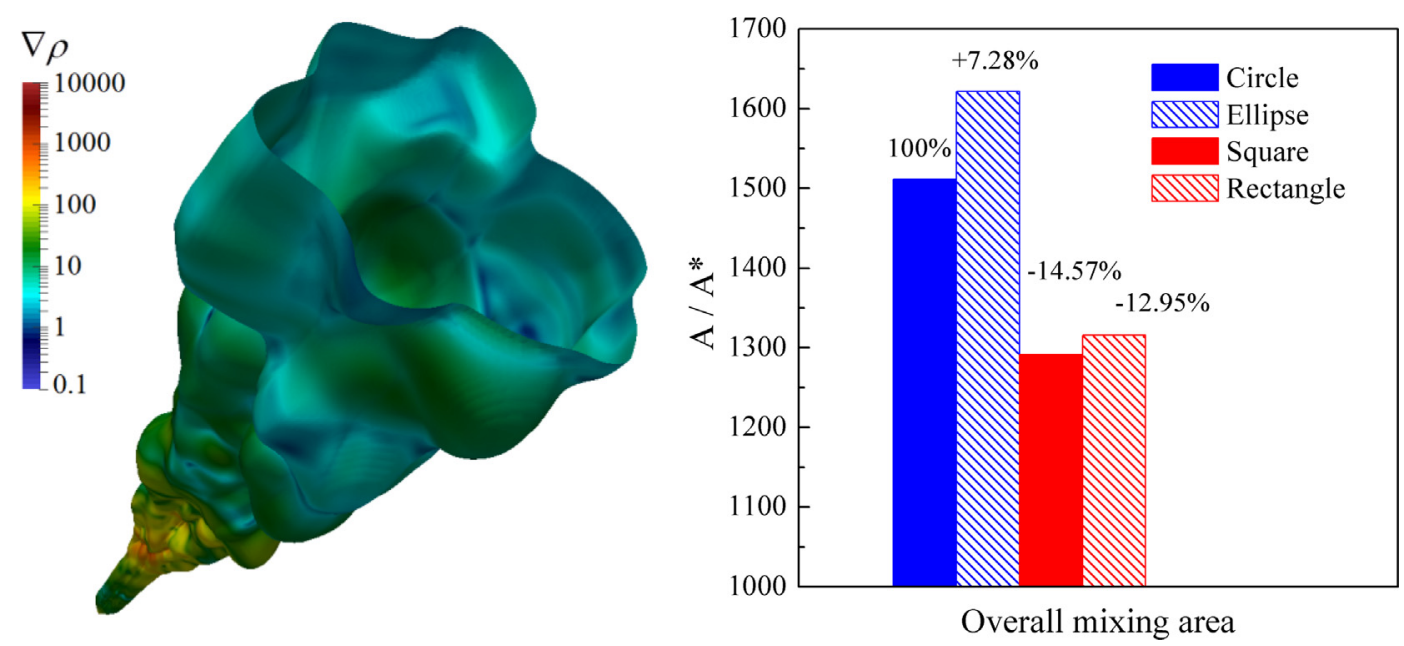

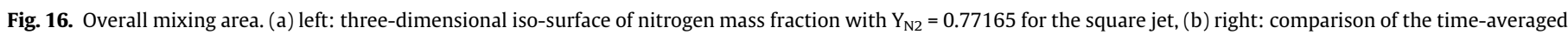
overall mixing area between different jets.

positions from $y / D=10$ to $y / D=28$, until decreases to about $11 \%$ at $\mathrm{y} / \mathrm{D}=30$.

Our previous study [35] indicates that the injection gas is still mixed with the ambient air at the jet boundary defined according to the mixing fraction level of $Z=0.02$. Fig. 16(a) shows the threedimensional iso-surface of nitrogen mass fraction for the square jet at $Y_{\mathrm{N} 2}=0.77165$, which corresponds to a mixture fraction level of $Z=0.02$. The area that the iso-surface covers can thus be used to evaluate the overall mixing characteristics of jets. Fig. 16(b) compares the time-averaged overall mixing area computed based on Fig. 16(a) for different jets. As can be seen, the overall mixing area of different jets has a similar profile as the mixing area on the cross-section planes as shown in Fig. 15(a), i.e., the elliptic jet has the largest overall mixing area, and the square jet has the smallest overall mixing area value, while the overall mixing area of the rectangular jet is larger than the square jet but smaller than the circular jet. However, it should be noted that the increment of the overall mixing area of the elliptic jet is $7.28 \%$ when compared to the circular jet, which is not as large as that indicated by Fig. 15. Similarly, the overall mixing area of the square and rectangular jets is $-14.57 \%$ and $-12.95 \%$ smaller than the circular jet, respectively.

\section{Conclusions}

In this study, large eddy simulations of highly underexpanded jets at NPR of 5.60 are carried out using an OpenFOAM solver for supersonic compressible flows, astroFoam. Four different nozzle geometries, i.e., the circular, elliptic, square, and rectangular nozzles, are employed to investigate the influence of the nozzle geometry on the flow behavior. Based on the results, the following concluding remarks are obtained:

(1) The flow evolution and development at different time is similar for different jets, and their turbulence transitions are all characterized by the breakdown of recirculation zones, the loss of flow symmetry, and the generation of streamwise vortexes. The square jet penetrates fastest and has a shortest FTT time of around $160 t_{0}$, while the elliptic jet penetrates slowest and takes a FTT time approaching to $260 \mathrm{t}_{0}$. The penetration rates of different jets show the similar linear dependency on the square root of time after the initial turbulent transition. The predicted penetration constant $\Gamma$ for the circular jet is 2.946, which is close to the theoretical value of 3.0. However, the penetration constants for other jets deviate more than $5 \%$ from 3.0, which suggests that the jet penetration constants $\Gamma$ are strongly influenced by the nozzle geometry used.

(2) The key flow features of the quasi-steady state square jet, including the shock structures, the acoustic patterns, and the jet boundary, are similar to the circular jet, and they both correspond to a three-dimensional helical instability mode. On the other hand, the flow characteristics of the elliptic jet are similar to the rectangular jet, but differ greatly from the circular and square jets. In particular, the large scale instability for the elliptic and rectangular jets is twodimensional in nature, and there is a lateral or flapping instability in the minor axis plane for these two jets. In addition, the shocks in the elliptic and rectangular jets oscillate wavily with larger amplitude in the minor axis plane, but appear to be steady and symmetric to the jet centerline in the major axis plane. Meanwhile, the jet boundary of the elliptic and rectangular jets expands extremely in the minor axis plane, but shrinks at first and then expands along the streamwise direction in the major axis plane.

(3) The predicted near-field shock structures in the circular jet agree well with the available literature data, and the smaller Mach disk diameter resolved by LES may be contributed to the differences in nozzle geometry design and simulation setup between the LES and experiment. The elliptic, square, and rectangular jets undergo a Mach reflection as the circular jet, but the Mach disk in the elliptic and rectangular jets is not easily visible due to its small size. The intercepting shock in the circular jet originates near the nozzle exit, and appears to be circular in cross-section. The intercepting shocks in the square jet originate at the four corners of the nozzle exit at first, and then are observed along the major axis plane some distance downstream of the nozzle exit. However, the formation of the intercepting shock is observed in the major axis planes but is lacking in the minor axis planes for the elliptic and rectangular jets. In addition, the axial locations of the shocks are similar for different jets, but the pressure in elliptic jet decreases to the ambient value more quickly.

(4) The mixing area on cross-section planes for different jets is relatively small in the near-field region, and increases quickly from around the end of jet potential core. Generally, the elliptic jet has the largest mixing area, and its mixing area takes an increment around 25\% from the end of jet 
potential core compared with the circular jet. The relative mixing area $\triangle A$ for the square and rectangular jets decreases along the streamwise direction, and reaches values below zero after around $\mathrm{y} / \mathrm{D}=10$, then approaches to a similar value of $-26 \%$ at $y / D=30$. The overall mixing area of the elliptic jet is largest as well, and is $7.28 \%$ larger than the circular jet. On the other hand, the overall mixing area of the square and rectangular jets is $-14.57 \%$ and $-12.95 \%$ smaller than the circular jet, respectively.

\section{Acknowledgments}

This project was funded by the National Natural Science Foundation of China (Grant Nos. 11502270 and 11602028) and China Postdoctoral Science Foundation (No. 2016M591258).

\section{References}

[1] V. Hindasageri, P. Kuntikana, A.R. Tajik, et al., Axis switching in impinging premixed methane-air flame jets, Appl. Therm. Eng. 107 (2016) 144-153.

[2] S. Biswas, S. Tanvir, H. Wang, et al., On ignition mechanisms of premixed $\mathrm{CH}_{4} /$ air and $\mathrm{H}_{2}$ /air using a hot turbulent jet generated by pre-chamber combustion, Appl. Therm. Eng. 106 (2016) 925-937.

[3] M. Berglund, E. Fedina, C. Fureby, J. Tegnér, Finite rate chemistry large-eddy simulation of self-ignition in supersonic combustion ramjet, AIAA J. 48 (3) (2010) 540-550.

[4] Z.P. Wang, F. Li, H.B. Gu, X.L. Yu, X.Y. Zhang, Experimental study on the effect of combustor configuration on the performance of dual-mode combustor, Aerosp. Sci. Technol. 42 (2015) 169-175.

[5] X. Liu, A. Godbole, C. Lu, G. Michal, P. Venton, Source strength and dispersion of CO2 releases from high-pressure pipelines: CFD model using real gas equation of state, Appl. Energy 126 (1) (2014) 56-68.

[6] X. Guo, X. Yan, J. Yu, Y. Zhang, et al., Under-expanded jets and dispersion in supercritical CO2 releases from a large-scale pipeline, Appl. Energy 183 (1) (2016) 1279-1291.

[7] J.A. Moríñigo, J.J. Salvá, Numerical study of the start-up process in an optimized rocket nozzle, Aerosp. Sci. Technol. 12 (6) (2008) 485-489.

[8] A. Hadjadj, Y. Perrot, S. Verma, Numerical study of shock/boundary layer interaction in supersonic overexpanded nozzles, Aerosp. Sci. Technol. 42 (2015) 158-168.

[9] E. Gutmark, F. Grinstein, Flow control with noncircular jets, Annu. Rev. Fluid Mech. 31 (1) (1999) 239-272.

[10] E. Rajakuperan, M.A. Ramaswamy, An experimental investigation of underexpanded jets from oval sonic nozzles, Exp. Fluids 24 (4) (1998) 291 299.

[11] D.M. Mitchell, D.R. Honnery, J. Soria, Near-field structure of underexpanded elliptic jets, Exp. Fluids 54 (7) (2013) 1-13.

[12] V. Chauhan, S.M.A. Kumar, E. Rathakrishnan, Mixing characteristics of underexpanded elliptic sonic jets from orifice and nozzle, J. Propul. Power 31 (2) (2015) 1-9.

[13] F. Grinstein, E. Gutmark, T. Parr, Near field dynamics of subsonic free square jets. A computational and experimental study, Phys. Fluids 7 (6) (1995) 1483 1497.

[14] F.F. Grinstein, C.R. Devore, Dynamics of coherent structures and transition to turbulence in free square jets, Phys. Fluids 8 (5) (1996) 1237-1251.

[15] E. Gutmark, K. Schadow, C. Bicker, On the near acoustic field and shock structure of rectangular supersonic jets, AIAA J. 28 (1) (1990) 1163-1170.

[16] G. Raman, R. Taghavi, Resonant interaction of a linear array of supersonic rectangular jets: an experimental study, J. Fluid Mech. 309 (1) (1996) 93-111.

[17] G. Raman, Screech tones from rectangular jets with spanwise oblique shockcell structures, J. Fluid Mech. 330 (1997) 141-168.

[18] P. Behrouzi, J.J. Mcguirk, Underexpanded jet development from a rectangular nozzle with aft-deck, AIAA J. 53 (5) (2015) 1287-1298.

[19] G. Valentich, P. Upadhyay, R. Kumar, Mixing characteristics of a moderate aspect ratio screeching supersonic rectangular jet, Exp. Fluids 57 (5) (2016) 1 14

[20] S.M.V. Rao, G. Jagadeesh, Novel supersonic nozzles for mixing enhancement in supersonic ejectors, Appl. Therm. Eng. 71 (1) (2014) 62-71.

[21] E. Gutmark, K.C. Schadow, K.J. Wilson, Noncircular jet dynamics in supersonic combustion, J. Propul. Power 5 (5) (1989) 529-533.

[22] E. Gutmark, K.C. Schadow, K.J. Wilson, Subsonic and supersonic combustion using noncircular injectors, J. Propul. Power 7 (2) (1991) 240-249.

[23] Y. Otobe, H. Kashimura, S. Matsuo, et al., Influence of nozzle geometry on the near-field structure of a highly underexpanded sonic jet, J. Fluids Struct. 24 (2) (2008) 281-293.
[24] H. Zare-Behtash, K. Kontis, N. Gongora-Orozco, et al., Compressible vortex loops: effect of nozzle geometry, Int. J. Heat Fluid Flow 30 (3) (2009) 561-576.

[25] H. Zare-Behtash, N. Gongora-Orozco, K. Kontis, Effect of primary jet geometry on ejector performance: a cold-flow investigation, Int. J. Heat Fluid Flow 32 (3) (2011) 596-607.

[26] N. Menon, B.W. Skews, Shock wave configurations and flow structures in nonaxisymmetric underexpanded sonic jets, Shock Waves 20 (3) (2010) 175-190.

[27] Xu et al., Effect of noncircular orifice plates on the near flow field of turbulent free jets, Chin. Phys. B 23 (12) (2014) 298-306.

[28] H. Zhang, Z. Chen, X. Jiang, et al., The initial flow characteristics of supersonic jets with different geometries, Phys. Lett. A 379 (18-19) (2015) 1256-1262.

[29] S. Tsutsumi, S. Teramoto, K. Yamaguchi, et al., Structure of underexpanded jets from square nozzles, AIAA J. 44 (6) (2006) 1287-1291.

[30] X. Yang, X. Long, X. Yao, Numerical investigation on the mixing process in a steam ejector with different nozzle structures, Int. J. Therm. Sci. 56 (2) (2012) 95-106.

[31] V. Vuorinen, J. Yu, S. Tirunagari, et al., Large-eddy simulation of highly underexpanded transient gas jets, Phys. Fluids 25 (1) (2013) 016101.

[32] V. Vuorinen, A. Wehrfritz, C. Duwig, B.J. Boersma, Large-eddy simulation on the effect of injection pressure and density on fuel jet mixing in gas engines, Fuel 130 (130) (2014) 241-250

[33] A. Hamzehloo, P.G. Aleiferis, Large eddy simulation of highly turbulent underexpanded hydrogen and methane jets for gaseous-fuelled internal combustion engines, Int. J. Hydrogen Energy 39 (36) (2014) 21275-21296.

[34] A. Hamzehloo, P.G. Aleiferis, Gas dynamics and flow characteristics of highly turbulent under-expanded hydrogen and methane jets under various nozzle pressure ratios and ambient pressures, Int. J. Hydrogen Energy 41 (15) (2016) 6544-6566.

[35] Xiaopeng Li, Wu. Kun, Wei Yao, Xuejun Fan, A comparative study of highly underexpanded nitrogen and hydrogen jets using large eddy simulation, Int. J. Hydrogen Energy 41 (9) (2016) 5151-5161.

[36] Xiaopeng Li, Wei Yao, Xuejun Fan, Large-eddy simulation of time evolution and instability of highly underexpanded sonic jets, AIAA J. 54 (10) (2016) 3191-3211.

[37] V. Chakravarthy, S. Menon, Large eddy simulations of turbulent premixed flames in the flamelet regime, Combust. Sci. Technol. 162 (2001) 175-222.

[38] M.W. Chase, JANAF thermochemical tables, J. Phys. Chem. Ref. Data 3 (2) (1974) 311-480

[39] A. Kurganov, E. Tadmor, New high-resolution central schemes for nonlinear conservation laws and convection-diffusion equations, J. Comput. Phys. 160 (1) (2000) 241-282.

[40] H. Jasak, H.G. Weller, A.D. Gosman, High resolution NVD differencing scheme for arbitrarily unstructured meshes, Int. J. Numer. Meth. Fluids 31 (2) (1999) 431-449.

[41] M.H. Baba-Ahmadi, G. Tabor, Inlet conditions for LES using mapping and feedback control, Comput. Fluids 38 (6) (2009) 1299-1311.

[42] C. Gorle, M. Gamba, F. Ham, Investigation of an underexpanded hydrogen jet in quiescent air using numerical simulations and experiments. Center for Turbulence Research Annual Research Briefs, Center for Turbulence Research, Stanford CA, 2010.

[43] A. Dauptain, B. Cuenot, Y.M. Gicquel, Large-eddy simulation of a stable supersonic jet impinging on flat plate, AIAA J. 48 (10) (2010) 2325-2337.

[44] Z.A. Rana, B. Thornber, D. Drikakis, Transverse jet injection into a supersonic turbulent cross-flow, Phys. Fluids 23 (2011) 046103.

[45] L.L. Yuan, R.L. Street, J.H. Ferziger, Large-eddy simulations of a round jet in crossflow, J. Fluid Mech. 379 (1999) 71-104.

[46] S. Drobniak, R. Klajny, Coherent structures of free acoustically stimulated jet, J. Turbul. 3 (2002) N1.

[47] P.G. Hill, P. Ouellette, Transient turbulent gaseous fuel jets for diesel engines, J. Fluids Eng. 121 (1999) 93-101.

[48] P. Ouellette, P.G. Hill, Turbulent transient gas injections, J. Fluids Eng. 122 (2000) 743-753.

[49] J. Panda, Shock oscillation in underexpanded screeching jets, J. Fluid Mech. 363 (1998) 173-198.

[50] D. Edgington-Mitchell, D.R. Honnery, J. Soria, The underexpanded jet Mach disk and its associated shear layer, Phys. Fluids 26 (2014) 096101.

[51] C.K.W. Tam, Supersonic jet noise, Annu. Rev. Fluid Mech. 27 (1995) 17-43.

[52] F. Hussain, H.S. Husain, Elliptic jets. Part 1. Characteristics of unexcited and excited jets, J. Fluid Mech. 208 (1989) 257-320.

[53] E. Franquet, V. Perrier, S. Gibout, et al., Free underexpanded jets in a quiescent medium: a review, Prog. Aerosp. Sci. 77 (2015) 25-53.

[54] A. Hamzehloo, P.G. Aleiferis, Numerical modelling of transient underexpanded jets under different ambient thermodynamic conditions with adaptive mesh refinement, Int. J. Heat Fluid Flow 61 (2016) 711-729.

[55] R.G. Panda, Seasholtz. Measurement of shock structures and shock-vortex interaction in underexpanded jets using Rayleigh scattering, Phys. Fluids 11 (12) (1999) 3761-3777.

[56] C.D. Donaldson, R.S. Snedeker, A study of free jet impingment. Part 1. Mean properties of free and impinging jets, J. Fluid Mech. 45 (1971) 281-319.

[57] S.B. Verma, E. Rathakrishnan, Effect of Mach number on the acoustic field of 2:1 elliptic-slot jet, Aeronaut. J. 105 (2001) 9-16. 